\title{
Nardilysin regulates axonal maturation and myelination in the central and peripheral nervous system
}

\section{AUTHOR(S):}

Ohno, Mikiko; Hiraoka, Yoshinori; Matsuoka, Tatsuhiko; Tomimoto, Hidekazu; Takao, Keizo; Miyakawa, Tsuyoshi; Oshima, Naoko; ... Kimura, Takeshi; Kita, Toru; Nishi, Eiichiro

\section{CITATION:}

Ohno, Mikiko ...[et al]. Nardilysin regulates axonal maturation and myelination in the central and peripheral nervous system. Nature Neuroscience 2009, 12: 1506-1513

\section{ISSUE DATE:}

2009-11-22

URL:

http://hdl.handle.net/2433/88956

\section{RIGHT:}

c 2009 Nature America, Inc. All rights reserved. 許諾条件により本文は 2010-05-23に公開.; This is not the published version. Please cite only the published version.; この論文は出版社版でありません。引用の際に は出版社版をご確認ご利用ください。 


\section{Nardilysin Regulates Axonal Maturation and Myelination in the Central and Peripheral Nervous System}

Mikiko Ohno ${ }^{1}$, Yoshinori Hiraoka ${ }^{1}$, Tatsuhiko Matsuoka ${ }^{1}$, Hidekazu Tomimoto ${ }^{2}$, Keizo Takao ${ }^{3,4,5}$, Tsuyoshi Miyakawa ${ }^{3,4,5}$, Naoko Oshima ${ }^{6}$, Hiroshi Kiyonari ${ }^{6}$, Takeshi Kimura $^{1}$, Toru Kita ${ }^{1,7}$, and Eiichiro Nishi ${ }^{{ }^{*}}$

${ }^{1}$ Department of Cardiovascular Medicine and ${ }^{5}$ Frontier Technology Center, Graduate School of Medicine, Kyoto University, 54 Shogoin-Kawahara-cho, Sakyo-ku, Kyoto 606-8507, Japan

${ }^{2}$ Department of Neurology, Graduate School of Medicine, Mie University, 2-174 Edobashi, Tsu 514-8507, Japan

${ }^{3}$ Devision of Systems Medical Science, Institute for Comprehensive Medical Science, Fujita Health University, 1-98 Dengakugakubo, Kutsukake-cho, Toyoake, Aichi 470-1192, Japan.

${ }^{4}$ Institute for Bioinformatics Research and Development and CREST, 4-1-8 Honcho, Kawaguchi, Saitama 332-0012, Japan.

${ }^{6}$ Laboratory for Animal Resources and Genetic Engineering, Center for Developmental Biology (CDB), RIKEN Kobe, 2-2-3 Minatojima Minami, Chuou-ku, Kobe 650-0047, Japan

${ }^{7}$ Present address: Kobe City Medical Center General Hospital, 4-6 Minatojima-nakamachi, Chuo-ku, Kobe 650-0046, Japan.

*Corresponding author

Correspondence should be addressed to E.N. (nishi@kuhp.kyoto-u.ac.jp). 
Axonal maturation and myelination are essential processes in establishing an efficient neuronal signaling network. Here, we show that nardilysin ( $\mathrm{N}$-arginine dibasic convertase: NRDc), a metalloendopeptidase enhancer of protein ectodomain shedding, is a critical regulator of these processes. $N R D c^{-1}$ mice had smaller brains with a thin cerebral cortex, in which there were less myelinated fibers with thinner myelin sheaths and smaller axon diameters. $N R D c^{-1-}$ mice also showed hypomyelination in the peripheral nervous system (PNS). Neuron-specific overexpression of NRDc induced hypermyelination, indicating that the level of neuronal NRDc regulates myelin thickness. Consistent with these findings, $\mathrm{NRDc}^{-/-}$ mice showed impaired motor activities and cognitive deficits. Further, NRDc enhanced ectodomain shedding of neuregulin1 (NRG1), which is a master regulator of myelination in the PNS. Based on these data, we propose that NRDc regulates axonal maturation and myelination in the CNS and PNS, in part, through the modulation of NRG1 shedding.

Myelination of axons by glial cells, such as oligodendrocytes in the CNS and Schwann cells in the PNS, is essential in rapid impulse conduction. Myelination is coordinated by the interaction between axons and glial cells. While the diameter of an axon dictates whether myelination is initiated, myelination may further induce radial growth of axons ${ }^{1,2}$. As axonal conduction is determined by axon caliber and myelin sheath thickness, axonal maturation (radial growth of axon) and myelination are essential processes for establishing an efficient neuronal signaling network ${ }^{3,4}$. Several lines of evidence suggest that bidirectional signaling between axon and myelin maintains neuronal functions. For instance, some oligodendrocyte-specific proteins, such as proteolipid protein and 2',3'-cyclic nucleotide phosphodiesterase (CNP), are required to maintain axonal integrity ${ }^{5,6}$. On the other hand, neuregulin1 (NRG1), a member of the epidermal growth factor (EGF) family, induces axonal signaling and is required for glial differentiation, proliferation and myelination ${ }^{7-9}$. NRG1 is synthesized 
as a transmembrane protein and then shed from the cell surface by proteolytic cleavage in the juxtamembrane region ${ }^{10}$. ADAM proteases and BACE1 ( $\beta$-secretase) have been proposed as sheddases for NRG111-15. As BACE1-deficient mice show a hypomyelination phenotype ${ }^{13-15}$, shedding of NRG1 is thought to be important in myelination in vivo. However, the underlying mechanism by which NRG1 shedding is regulated is poorly understood.

NRDc is a zinc peptidase of the M16 family, which selectively cleaves dibasic sites $^{16,17}$. We identified NRDc as a specific binding partner of HB-EGF, and demonstrated that NRDc enhances HB-EGF shedding through activation of tumor necrosis factor- $\alpha$ converting enzyme (TACE; ADAM17) ${ }^{18,19}$. We also showed that NRDc enhances the ectodomain shedding of multiple membrane proteins including amyloid precursor protein (APP) and tumor necrosis factor-a through activation of several ADAM proteases ${ }^{20,21}$. These results suggest that NRDc may regulate the ectodomain shedding of a wide range of membrane proteins. To explore the physiological functions of NRDc, we generated NRDc-deficient mice and found that these mice show impaired axonal maturation and hypomyelination in both the CNS and PNS. Further, we found that NRDc regulates axonal maturation and myelination in the CNS and PNS, in part, through the modulation of NRG1 shedding. 


\section{RESULTS}

\section{Small brains in $N R D c^{-/-}$mice}

NRDc is highly expressed in brain lysates of early postnatal mice, but at a lower level in those of adult mice (Fig. 1a; left panel). Immunohistochemical analysis showed that NRDc is expressed in neurons and dendrites, but not in glial cells (Fig. 1b and Supplementary Fig. 4a) ${ }^{20}$. These findings were supported by in situ hybridization, which showed little or no $N R D c$ mRNA in the corpus callosum (Supplementary Fig. 3).

To examine the physiological functions of NRDc, we generated mice deficient in NRDc by gene targeting (Supplementary Fig.1a). No $N R D c$ mRNA or protein was detected by real-time PCR (data not shown) and Western-blot analysis, respectively, in the brain (Fig. 1a; center panel) and all other tissues tested from $N R D c^{-/-}$mice (data not shown). Immunostaining of tissue sections also showed a lack of NRDc protein in the cerebral cortex of $N R D c^{-/}$mice (Supplementary Fig. 1c). Levels of NRDc protein in embryonic fibroblasts (MEF) isolated from $N R D c^{+/+}, N R D c^{+/-}$, or $N R D c^{-/}$mice correlated with the predicted gene dosage of $N R D c$ in these mice (Fig. 1a; right panel).

Pups lacking NRDc were born at the expected Mendelian ratio. However, approximately $80 \%$ died within 48 hours after birth. $N R D c^{-/-}$pups weighed approximately $30 \%$ less than $N R D c^{+/+}$and $N R D c^{+/-}$littermates, indicating that NRDc is indispensable for normal prenatal growth. $N R D c^{-/}$mice that survived remained smaller than their wild-type and heterozygous littermates throughout postnatal development, and had average brain weights $29 \%$ below those of $N R D c^{+/+}$mice at postnatal day 90 (P90) $\left(N R D c^{+/+} ; 521.0 \pm 30.0 \mathrm{mg}, N R D c^{-/-} ; 371.5 \pm 16.8 \mathrm{mg}, \mathrm{n}=5, \mathrm{p}<0.001\right)$ (Fig. 1c). While $N R D c^{-/-}$mice that survived lived until two years of age, they showed several prominent neurological disorders. For example, $N R D c^{-/}$mice exhibited enhanced limb-clasping reflexes when suspended by the tail, whereas control mice extended their limbs (Fig. 1d). 
To assess the underlying neuropathology, brains of $N R D c^{-/-}$mice were examined histologically. While the gross anatomy of $N R D c^{-/-}$brain was normal at P1, P14 and P30 (Supplementary Fig. 2), a thin cerebral cortex and enlarged lateral ventricles were observed at P90 (Fig. 1e). Nissl staining of the $N R D c^{-/}$cortex demonstrated greater neuronal cell density than in the $N R D c^{+/+}$cortex (Fig. 1f,g). Furthermore, there were no differences in the number of TUNEL-positive cells detected in the cortex of $N R D c^{+/+}$and $N R D c^{-/}$mice (data not shown), indicating no excessive loss of neurons in the $N R D c^{-/}$cortex.

\section{Hypomyelination in the CNS of $N R D c^{-/-}$mice}

We next examined the integrity of axons and myelin using silver impregnation and luxol fast blue (LFB) staining, respectively. In $N R D c^{-/-}$brain, much less silver impregnation was detected, especially in the corpus callosum and cortical layers adjacent to it (Fig. $\mathbf{2 a , c})$. Similarly, LFB staining was markedly weaker in this region (Fig. 2b,d). These results suggested a decreased volume of axons and myelin in $N R D c^{-/}$brain, especially in the corpus callosum. Furthermore, levels of the axonal marker neurofilament-H (NF-H; Fig. 2e), and myelin markers, CNP and myelin basic protein (MBP) (Fig. 2f), were lower in the corpus callosum in $N R D c^{-/-}$brain at P30. Western blotting also showed reduced CNP and MBP protein levels even at an earlier stage (P14) in NRDc ${ }^{-/}$ brain extracts (Fig. 2g), suggesting that $N R D c^{-/-}$mice manifest a severe impairment of axonal maturation and hypomyelination starting at the early stages of these processes. Neuronal expression of NRDc was detected in whole regions of the brain, but there were some prominent regional differences. For example, NRDc was highly expressed in cortical neurons, but neurons in striatum expressed relatively low levels of NRDc (Supplementary Fig. 4a), similar to the expression pattern in human brain ${ }^{22}$. Consistent with these results, the myelination defect in $\mathrm{NRDC}^{-/-}$striatum was not as obvious as the defect in the cortex, hippocampus and corpus callosum (Supplementary Fig. 4b). 
These results suggest a correlation between the expression level of NRDc and the extent of myelination defect in $N R D c^{-/-}$mice.

On the other hand, Western blotting using the same set of brain extracts showed that the expression of platelet-derived growth factor receptor- $\alpha$ (PDGFR- $\alpha$ ) and oligodendrocyte lineage gene 2 (olig2) ${ }^{23}$, markers of oligodendrocyte-precursors, and GST- $\pi$, a marker of mature oligodendrocyte, are not reduced in $N R D c^{-/}$(Fig. 2g), suggesting that oligodendroglial differentiation is normal in $\mathrm{NRDC}^{-/}$mice. Moreover, quantitative analysis of olig2-positive cells at E18.5 and P5 demonstrated that there are no differences in the number of oligodendrocyte precursors between $N R D c^{+/+}$and $N R D c^{-/-}$brains (Supplementary Fig. 5). These data suggest that NRDc affects myelination, but not differentiation of oligodendrocytes.

To obtain direct evidence of impaired axonal maturation and hypomyelination in the CNS, we analyzed sections of the corpus callosum in $N R D c^{-/-}$mice and $N R D c^{+/+}$ littermates between P14 and P365 by electron microscopy (Fig. 3a, and Supplementary Fig. 6a). The proportion of myelinated axons was dramatically reduced in $N R D c^{-/}$mice at all stages examined (Fig. 3c). At P14, only approximately one seventh of $N R D c^{-/}$axons were myelinated compared to $N R D c^{+/+}$axons, indicating a significant delay in the initiation of myelination in $N R D c^{-/-}$mice. The proportion of myelinated axons in the $N R D c^{-/-}$CNS was only $21.0 \%$ at P90, and did not approach the level seen in $N R D c^{+/+}$mice even at P365 (Fig. 3c). We next compared myelin sheath thickness in the $N R D c^{-/-}$and $N R D c^{+/+}$corpus callosum by determining the g-ratio ${ }^{24}$ (axon diameter / total fiber diameter) of myelinated fibers at P30, P90, P120 and P365 (Fig. 3b, and Supplementary Fig. 6b). Average g-ratios were significantly higher in $N R D c^{-/-}$mice at all stages (Fig. 3d), indicating that myelin sheaths are thinner in $N R D c^{-/-}$compared to those of $N R D c^{+/+}$mice. The average diameters of myelinated axons were also significantly smaller in $N R D c^{-/-}$mice than wild-type littermates (Fig. 3e). Although axons were hypomyelinated, the ultrastructure of myelin sheaths of 
$N R D c^{-/-}$mice appeared normal and indistinguishable from those of $N R D c^{+/+}$mice (data not shown). Together, these data indicate that $N R D c^{-/}$mice have fewer myelinated fibers in the corpus callosum, and the fibers have thinner myelin sheaths and smaller axon diameters. As NRDc is highly expressed in neurons, but not expressed or expressed at very low levels in glial cells (Fig. 1b, Supplementary Fig. 4a) ${ }^{20}$, we propose that the loss of NRDc in neurons may cause an impairment of axonal maturation, resulting in perturbation of axonal signaling required for oligodendrocytes to properly myelinate axons and hypomyelination.

\section{Hypomyelination in the PNS of $N R D c^{--}$mice}

NRDc is also expressed in spinal neurons and dorsal root ganglion neurons (Supplementary Fig. 3b). To investigate the role of NRDc in axonal maturation and myelination in the PNS, we analyzed sciatic nerves of $N R D c^{-/-}$mice by electron microscopy and found that sciatic nerve of $N R D c^{-/}$mice is hypomyelinated (Fig. 4a). Quantitative analysis by measuring the g-ratio of myelinated fibers demonstrated that the myelin sheath thickness is thinner in $N R D c^{-/}$mice at both P30 and P365. The diameter of myelinated axons was also significantly smaller in $N R D c^{-/}$nerve compared with $N R D c^{+/+}$nerve (Fig. 4b,c).

We also examined the morphology of Remak bundles (Fig. 4d,e,f). Remak bundles in $N R D c^{-/}$nerves contained approximately two times more axons than $N R D c^{+/+}$nerves at P30 and P365 (Fig. 4d,e). In addition, there were many unsegregated or poorly-segregated axons which lacked intervening Schwan cell processes in $\mathrm{NRDC}^{-/-}$ bundles (Fig. 4d,f). These findings indicate that NRDc is critical in axonal maturation and myelination of both the CNS and PNS. Interestingly, the observed hypomyelination and morphological change of Remak bundles in the PNS of $N R D c^{-/}$mice were very

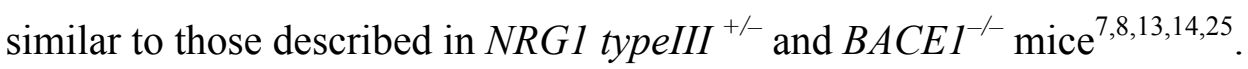




\section{Level of neuronal NRDc regulates myelin thickness}

To determine the dose-dependent effects of NRDc expression on the CNS phenotypes, we examined brains from $N R D c^{+/-}$mice. The gross anatomy of $N R D c^{+/-}$brains showed no cortical shrinkage, but did show significantly enlarged lateral ventricles (Fig. 5a). While no obvious differences between $N R D c^{+/+}$and $N R D c^{+/-}$brains were detected by LFB staining (Fig. 5a,b), immunostaining showed decreased expression of the myelin and axon markers, MBP and NF-H, in $N R D c^{+/-}$corpus callosum (Fig. 5d,e). These findings were confirmed by Western blotting using whole brain lysates (Fig. 5f). Further, we analyzed $N R D c^{+/}$corpus callosum by electron microscopy and found that the myelin sheath thickness of $N R D c^{+/-}$mice is significantly thinner than that of $N R D c^{+/+}$ mice (Average g-ratio, $N R D c^{+/+} ; 0.725 \pm 0.020$ s.e.m., $N R D c^{+/-} ; 0.761 \pm 0.001$ s.e.m., $\mathrm{n}$ $>1000$ fibers from each group, $\mathrm{P}<0.0001$; Fig. 5g,h). Axon diameters of $N R D c^{+/-}$mice were also smaller compared to those of $N R D c^{+/+}$mice $\left(N R D c^{+/+} ; 0.650 \pm 0.018 \mu \mathrm{m}\right.$, $\left.N R D c^{+/-} ; 0.582 \pm 0.006 \mu \mathrm{m}, \mathrm{P}<0.0001\right)$. Thus, $N R D c^{+/-}$mice show an intermediate CNS phenotype between $N R D c^{+/+}$and $N R D c^{-/-}$mice, indicating that the level of NRDc expression in neurons affects axonal maturation and myelination.

The loss-of-function approach using $N R D c^{-/-}$mice revealed that NRDc is essential for axonal maturation and myelination in the CNS. To further define the role of NRDc in these processes, a gain-of-function approach using transgenic mice that overexpress mouse NRDc under the control of the CamKII promoter (NRDc-Tg) was undertaken $^{26,27}$. Western blot analysis of whole brain extract of NRDc-Tg mice at P90 showed increased NRDc expression (Fig. 6a). The CamKII promoter is specifically activated in neurons of cerebral cortex and hippocampus ${ }^{26,27}$, where the increased expression of NRDc was confirmed by immunostaining (Fig. 6b,c). To see the regional effect of NRDc overexpression, we divided mouse brains into two regions: cerebral cortex plus hippocampus (frontal region) and brain stem plus cerebellum (posterior region). Western immunoblotting showed increased expression of NRDc protein only in 
the frontal region (Fig. 6d). The expression of myelin markers, MBP and CNP, was also increased in the frontal region, but not in the posterior region (Fig. 6d). Higher expression of MBP in corpus callosum was also detected by immunostaining (Fig. 6e). Importantly, analysis of myelination in the NRDc-Tg corpus callosum by electron microscopy demonstrated that the average g-ratio was significantly lower, indicating that the myelin sheaths are thicker in NRDc-Tg mice (Fig. 6g,h). On the other hand, there was no significant difference in the diameter of myelinated axons in the corpus callosum between the NRDc-Tg and wild-type mice (average diameter, wild-type; 0.80 $\pm 0.39 \mu \mathrm{m}, \mathrm{NRDc}-\mathrm{Tg} ; 0.81 \pm 0.39 \mu \mathrm{m}, \mathrm{n} . \mathrm{s})$. These results provide further evidence that the level of neuronal NRDc regulates myelin sheath thickness. The CamKII promoter is activated around P5, which is after the differentiation of neuronal cells and prior to subcortical myelination ${ }^{27}$. Thus, NRDc most likely affects myelination through regulating axonal signals transmitted from differentiated neurons.

\section{Impaired motor activity and cognitive deficits in $N R D c^{-}-$mice}

To examine the effect of NRDc on neurological functions, $N R D c^{-/-}$mice were analyzed with a battery of behavioral tests ${ }^{28}$. Neuromuscular strength was examined with grip strength and wire-hanging tests. While $N R D c^{-/-}$mice showed reduced grip strength (Fig. 7a), no significant difference between $N R D c^{+/+}$and $N R D c^{-/-}$mice was detected in the wire-hanging test (Supplementary Fig. 7a), indicating that $N R D c^{-/-}$mice possess sufficient grip strength to hold their body weight. We next assessed motor coordination and balance with the beam test and rotarod test $\mathrm{t}^{29}$. In the beam test, $N R D c^{-/-}$mice moved much slower and slipped more frequently than $N R D c^{+/+}$mice (Fig. 7b,c). $N R D c^{-/-}$mice also had a significantly shorter latency to fall in the rotarod test (Fig. 7d). These results indicate that there is a severe impairment of motor coordination and balance in the $N R D c^{-/-}$mice.

In contrast to motor activity, sensory pathways are apparently conserved in 
$N R D c^{-/-}$mice because their reactions were similar to those of $N R D c^{+/+}$mice in the hot plate test (Supplementary Fig. 7b). Cognitive functions of $N R D c^{-/}$mice were examined with the T-maze test, in which working memory and reference memory were evaluated by a forced alternation task and a left-right discrimination task, respectively ${ }^{30}$. In both tasks, $N R D c^{-/-}$mice took much longer to complete a session, most likely due to impaired motor activity (Supplementary Fig. 7c and data not shown). While the percentage of correct choices in the forced alternation task was significantly lower in $N R D c^{-/-}$mice, there were no significant differences in the left-right discrimination task between $N R D c^{+/+}$and $N R D c^{-/-}$mice (Fig. 7e,f). Together, these results demonstrate that the reference memory is preserved, whereas the working memory is impaired in $N R D c^{-/}$mice. While these behavioral alterations are consistent with the axonal and myelination defects, spinal or cerebellar defects might also influence the overall performance of $N R D c^{-/-}$mice.

\section{NRDc regulates NRG1 shedding through BACE1 and TACE}

NRG1, one of the master regulators of myelination, is synthesized as a transmembrane protein and then proteolytically shed from the cell surface $e^{8,10}$. Recent reports of hypomyelination in BACE1-deficient mice have implicated BACE1 in NRG1 shedding ${ }^{13-15}$. NRG1 is also shed from the cell surface by TACE ${ }^{11}$, although there are no information about nervous system phenotypes of TACE-deficient mice due to its early lethality. These findings, along with the potentiating effect of NRDc on protein ectodomain shedding ${ }^{19-21}$, prompted us to examine whether NRDc affects axonal maturation and myelination through regulating NRG1 shedding by BACE1 or TACE. To determine whether NRDc potentiates BACE1/TACE activity, we performed co-transfection experiments in COS7 cells. NRG1 type I was tagged with HA at the N-terminus, so that the full length and N-terminal fragment (NTF) of NRG1 type I could be detected with an anti-HA antibody. Co-expression of NRDc and BACE1 
increased NTF levels in total cell lysates, but not in the conditioned medium (Fig. 8a,b). In contrast, co-expression of NRDc with TACE clearly increased NTF levels in the conditioned medium (Fig. 8a,b). These results suggest that NRDc potentiates TACE-mediated NRG1 cleavage at the cell surface, whereas NRDc enhances BACE1 cleavage of NRG1 in intracellular compartments. As the enhancement of BACE1-mediated cleavage of NRG1 was not associated with an increase in NTF levels in the conditioned medium, NRDc is thought to potentiate BACE1 in endocytic pathways and direct the cleaved NRG1 to degradation ${ }^{31}$. Similar to the direct interaction of NRDc and TACE ${ }^{19}$, co-precipitaion demonstrated that NRDc forms as trimolecular complex with BACE1 and NRG1, suggesting that these proteins physically and functionally interact (Fig. 8c).

Given these findings, we examined the expression pattern of endogenous NRG1 protein in fibroblasts derived from $N R D c^{-/-}$and $N R D c^{+/+}$mice. Western blot analysis using the antibody against the C-terminus of NRG1 (NRG-C), which recognizes both full length NRG1 and C-terminal fragment of NRG1 (CTF), showed an increase in full length NRG1 and decrease in $\mathrm{CTF}$ in $N R D c^{-/-}$cells compared to $N R D c^{+/+}$cells $($Fig. 8d; left). In addition, while an antibody against the intracellular N-terminus of NRG1 type III detected the cleaved NTF of NRG1 type III in NRDc ${ }^{+/+}$ cells, it barely detected the cleaved fragment in $N R D c^{-/}$cells (Fig. 8d; right). These results indicate that NRDc positively regulates the proteolytic cleavage of both type I and type III NRG1.

Next, we examined the expression of NRG1 in brain extracts from $N R D c^{+/+}$ and $N R D c^{-/-}$mice at P14. Western blotting with antibodies against the C-terminus of NRG1 type I and N-terminus of NRG1 type III showed a similar pattern as found in MEF cells (Fig. 8e), although the difference between the two genotypes was less evident in the brain extract. These results are consistent with the fact that the proteolytic cleavage of NRG1 type I and type III in brain is regulated by NRDc. We then analyzed 
the protein expression of BACE1. Intriguingly, mature BACE1 in $N R D c^{-/-}$brain was a little smaller than that of $N R D c^{+/+}$brain (Fig. 8e). In addition, the ratio of the mature form to immature form of BACE1 was clearly higher in $N R D c^{+/+}$brains compared with $N R D c^{-/}$brains $\left(N R D c^{+/+} ; 2.21 \pm 0.62, N R D c^{-/-} ; 0.75 \pm 0.08, \mathrm{n}=3, \mathrm{P}=0.04\right)$, indicating that the protein maturation of BACE1 is impaired in $N R D c^{-1-}$ brains. These results suggest that NRDc regulates BACE1-mediated NRG1 shedding through affecting BACE1 maturation and BACE1 sheddase activity. On the other hand, the mRNA levels of NRG1 and BACE1 were not significantly different between $N R D c^{+/+}$and $N R D c^{-/-}$ brains (Supplementary Fig. 8), further indicating that NRDc post-translationally modulates the expression and activity of NRG1 and BACE1. 


\section{DISCUSSION}

Our findings provide the first in vivo evidence that NRDc is a critical regulator of axonal maturation and myelination in the CNS and PNS. In the CNS, axon diameter and myelin thickness correlated with levels of NRDc expression (homozygous < heterozygous < wild-type mice). Our results suggested that NRDc affects myelination through regulating axonal maturation because myelin thickness was proportional to the diameter of axons ${ }^{1,2}$. Neuron-specific overexpression of NRDc, however, did not affect the axon caliber, but instead induced hypermyelination, providing additional evidence that NRDc is a critical regulator of myelination. Importantly, because we did not see any differences in the number of oligodendrocyte precursor cells and the expression level of specific oligodendrocyte precursor markers between $N R D c^{+/+}$and $N R D c^{-/-}$ mice, NRDc appears to specifically regulate myelination, and not oligodendrocyte differentiation nor proliferation. In addition, as expression of NRDc is confined to neurons, the effect of NRDc on oligodendrocyte is non-cell autonomous. Instead, our data suggest that NRDc affects axonal signaling between neurons and oligodendrocytes.

NRG1 is the best characterized neuronal factor that induces axonal signaling required for the entire program of glial differentiation, proliferation and myelination $^{8,10,32}$. NRG1, a member of EGF family, is synthesized as a transmembrane protein that is proteolytically cleaved in the juxtamembrane region. ADAM proteases (TACE / ADAM17 $7^{11}$ and ADAM1912,33) and BACE $1^{13,14}$ have been proposed to cleave NRG1. Further, BACE1-deficient mice exhibit hypomyelination ${ }^{13-15}$ and ADAM19-deficient mice show delayed remyelination after injury ${ }^{33}$, suggesting that proteolytic cleavage of NRG1 is important for myelination. Our data indicate that NRDc is a critical regulator of NRG1 cleavage in vivo. First, gain of function experiments in cells showed that NRDc enhances BACE1- and TACE-mediated NRG1 cleavage. Second, loss of function experiments in cells showed an increase of the full-length NRG1 and a decrease of cleaved NRG1, indicating that NRG1 cleavage is 
reduced in the absence of NRDc. Further, similar to the results from $N R D c^{-/-}$cells, brain lysates of $N R D c^{-/}$mice showed a significant decrease in levels of cleaved NRG1. $\mathrm{NRDC}^{-/-}$mice also showed hypomyelination not only in the CNS, but also in the PNS, where NRG1 is a master regulator of myelination. Lastly, we showed that NRDc forms a complex with BACE1 and NRG1, suggesting that these molecules interact functionally. The present study, therefore, suggest that regulation of NRG1 proteolysis by NRDc is a critical post-translational modification for myelination.

Although several studies have suggested the involvement of NRG1 in oligodendrocyte differentiation and CNS myelination ${ }^{25,32,34}$, the role of NRG1 in CNS myelination is still controversial due to the embryonic lethality of NRG1 deficient mice. However, a recent study showed normal myelination in a series of conditional null mutants of NRG1 that lack the gene at different stages during CNS development, suggesting that NRG1 may function differently in the PNS and $\mathrm{CNS}^{35}$. Because our data indicated that NRDc regulates myelination in both the CNS and PNS, NRDc may affect different proteins in the CNS. On the other hand, while both $\mathrm{NRDc}^{-/}$and $B A C E 1^{-/}$ mice showed hypomyelination in the CNS and PNS, no abnormalities in axon diameter and initiation of myelination have been reported in $B A C E 1^{-/}$mice $^{13-15}$. Taken together, the effect of NRDc in the CNS cannot be attributed merely to NRG1 or BACE1. We have previously shown that NRDc potentiates several ADAM proteases ${ }^{19-21}$ and show here that NRDc also enhances the capacity of BACE1 to process NRG1. The enhancing effect of NRDc on ADAM proteases is not substrate-specific because NRDc can potentiate TACE-mediated shedding of HB-EGF, TNF- $\alpha$, APP and NRG1 ${ }^{19-21}$. This might also be the case for BACE1 because our studies showed that NRDc affects the maturation of BACE1 (Fig. 8).

Axonal maturation and myelination are essential in nerve conduction and aberrant in various neuropathologies. For example, the primary pathologic event of multiple sclerosis is demyelination, and remyelination correlates with recovery from 
clinical symptoms ${ }^{36}$. Given the dose-dependent effect of NRDc on myelin thickness, NRDc could be a potential pharmacological target of this most common neurological disorder of young adults. NRDc could also play an important role in the regeneration of injured axons. In fact, the critical regulatory functions of NRDc in axon-oligodendrocyte signaling, axon maturation and myelination suggest that NRDc may impinge on a broad range of neurological disorders. 


\section{Acknowledgement}

We are grateful to N. Nishimoto and H. Nakabayashi for technical assistance, and K. Matsumoto, E. Kimura, A. Kinoshita and A. Sehara for materials. We thank P.W. Park, T. Nishio, H. Fujiwara, T. Kaneko, F. Fujiyama and H. Kawasaki for critical reading of the manuscript. This study was supported by Research grants (19041035, 20390255, 20659061 and 20200068) from the Ministry of Education, Culture, Sports, Science and Technology of Japan. It was also supported by the Takeda Science Foundation, the Mochida Memorial Foundation for Medical and Pharmaceutical Research, the Suzuken Memorial Foundation, the Japan Health Foundation and the Daiichi Sankyo Sponsored Research Program. The authors declare no competing financial interests.

\section{Author Contribution}

M.O. and E.N. planned experiments and wrote the manuscript. M.O., Y.H., T.M. and E.N. performed experiments. H.T. performed histological procedures. K.T. and T.M. performed behavioral analysis. N.O. and H.K. generated NRDc-deficient mice. T.Kimura and T.Kita supervised the work. 


\section{Figure Legends}

Figure 1 Expression of NRDc in brains and gross CNS phenotypes of $\mathrm{NRDc}^{-/-}$mice. (a) Immunoblot analysis of brain extracts (left, center) or total cell extracts (right) stained with antibodies to NRDc and GAPDH. Analysis of brain extracts from wild-type mice at 5, 14, 90, and 365 postnatal days (P5, P14, P90 and P365; left); and from control wild-type $(+/+)$ and $N R D c^{-/-}$mice $(-/-)$at P90 (center). Analysis of the total extracts of MEF isolated from $N R D c^{+/+}, N R D c^{+/-}$, or $N R D c^{-/-}$mice (right). Full -length blots are presented in Supplementary Figure 9. (b) Immunohistochemistry using an anti-NRDc antibody (green) with cerebral cortex sections from wild-type mice at P30. The section was double-stained with fluorescent Nissl (red). Scale bars, $250 \mu \mathrm{m}$. (c) Brains of $N R D c^{-/-}$mice (right) and control wild-type littermates (left) at P90. (d) Enhanced limb-clasping reflex of $N R D c^{-/}$(right) compared with wild-type (left) mice at P42. (e) H\&E-stained sections of the brain hemisphere of $N R D c^{-/-}$mice (right) and wild-type littermates (left) at P90. An enlargement of the lateral ventricles and cortical shrinkage in $N R D c^{-/}$brain is shown. (f,g) Nissl-stained sections of cerebral cortex of $N R D c^{-/-}$mice (right) and wild-type littermates (left) at P90 (Scale bars, $500 \mu \mathrm{m}$ ). Counting of Nissl-positive neurons at the same bregma levels (6 non-overlapping fields $/ \mathrm{n}=2$ per genotype, results are mean \pm s.e.m. ${ }^{*} \mathrm{p}<0.01$ ) showed greater neuronal cell density in the $N R D c^{-/}$cortex.

Figure 2 Impaired axonal maturation and hypomyelination in the CNS of $N R D c^{-/}$mice. (a-d) Silver impregnation by Bielschowskey method $(\mathbf{a}, \mathbf{c})$ and LFB staining $(\mathbf{b}, \mathbf{d})$ of $N R D c^{-/-}$brain (-/-; right) and wild-type brain (+/+; left) at P90, showing impairment of axonal maturation and hypomyelination in $N R D c^{-/}$brain (a,b; Scale bars, $2 \mathrm{~mm}$ ), especially in the corpus callosum (c,d; Scale bars, $500 \mu \mathrm{m})$. (e,f) Immunohistochemistry using anti-NF-H antibody (e), anti-CNP antibody and anti-MBP antibody (f) of corpus callosum from control wild-type mice $\left(+/+\right.$; left) and $N R D c^{-/-}$mice $(-/-$; right) at P30. 
Scale bars, e; $250 \mu \mathrm{m}, \mathbf{f} ; 500 \mu \mathrm{m}$. (g) Immunoblot analysis of brain extracts from wild-type and $\mathrm{NRDc}^{-/}$brains at P14 showed a reduction of MBP, CNP and NF-H expression in $\mathrm{NRDc}^{-/-}$brains. On the other hand, there were no differences in Olig2, PDGFR- $\alpha$, and GST- $\pi$, marker proteins of oligodendrocyte precursor or mature oligodendrocyte ( $\mathrm{n}=3$ in each genotype). Full-length blots are presented in Supplementary Figure 9.

Figure 3 Delay in the initiation of myelination and hypomyelination in $N R D c^{-/}$mice. Electron microscopic analysis of corpus callosum. (a) Electron micrographs of transverse sections at the corpus callosum from $N R D c^{-/-}$mice (right) and wild-type littermates (left) at P14 (upper) and P90 (bottom). Scale bars, $2 \mu \mathrm{m}$. (b,d) Quantitation of myelin sheath thickness in the corpus callosum by analyzing g-ratios with electron micrographs. Scatter plots of myelin thickness, expressed as g-ratios, against axon diameters at P90 and P365 in $N R D c^{-/}$(black) and wild-type (red) corpus callosum (b). Average myelin sheath thickness (g-ratio) of myelinated fibers at P30, P90, P120 and P365, showing quantitative evidence of hypomyelination in the corpus callosum of $N R D c^{-/-}$mice $(\mathbf{d})$. Data represent the mean \pm s.e.m. for 300 to 900 myelinated fibers from each group. ${ }^{*}, \mathrm{p}<0.03, * *, \mathrm{p}<0.0001$. (c) Percentage of myelinated axons in the corpus callosum of $N R D c^{-/}$(black bars) and wild-type littermates (white bars) at P14, P30, P90, and P365. (e) Average diameters of myelinated axons in the corpus callosum at P30 and P365. Data represent the mean \pm s.e.m. for 300 to 900 myelinated fibers from each group. ${ }^{*}, \mathrm{p}<0.0003, * *, \mathrm{p}<0.0001$.

Figure 4 Impaired axonal maturation and hypomyelination in the PNS of $N R D c^{-/-}$mice. (a) Electron micrographs of sciatic nerves of $N R D c^{+/+}$(left) and $N R D c^{-/-}$(right) mice at P365 showed obvious hypomyelination in $N R D c^{-/-}$nerve. Scale bars; $2 \mu \mathrm{m}$. (b) Scatter plots of g-ratios and axon diameters at P30 and P365 of $N R D c^{-/-}$(black) and wild-type 
(red) sciatic nerves. (c) G-ratios and axon diameters at P30 and P365 of $N R D c^{+/+}$and $N R D c^{-/-}$sciatic nerves. Data represent the mean \pm s.e.m. for 750 myelinated fibers from each group $(\mathrm{n}=4$, for each genotype). *; $\mathrm{p}<0.03$, **; $\mathrm{p}<0.01$. (d) Electron micrographs of Remak bundles in sciatic nerves show altered axonal segregation in $N R D c^{-/-}$bundles. Arrows indicate Schwann cell cytoplasmic processes between different axons. Note that many axons of $N R D c^{-/-}$bundles are directly apposed without the cytoplasmic processes (arrowhead). Scale bars; $500 \mathrm{~nm}$. (e) Average numbers of axons in a Remak bundle at P30 and P365 were significantly reduced in $N R D c^{-/-}$sciatic nerves. Data represent the mean \pm s.e.m. for 150 to 300 Remak bundle from each group ( $\mathrm{n}=2$ for each genotype). ${ }^{* * *} ; \mathrm{p}<0.0001$. (f) Ratios of segregated axons in $N R D c^{-/-}$ sciatic nerves at P30 and P365 were reduced (150 to 300 Remak bundle from each group, $\mathrm{n}=2$ for each genotype).

Figure 5 Intermediate CNS phenotype in heterozygous mutant of NRDc-deficient mice. (a,b) LFB staining of $N R D c^{+/-}$brains (right) showed enlarged lateral ventricles especially in the frontal section (a), compared with wild-type brains (left), but no obvious cortical shrinkage. Scale bars, $1 \mathrm{~mm}$. (c) Immunohistochemistry of $N R D c^{+/}$ cortex (+/-; bottom) with anti-NRDc antibody showed the obvious reduction of NRDc expression. (d,e) Immunohistochemistry of $N R D c^{+/+}$(left) and $N R D c^{+/-}$(right) corpus callosum at P90 using anti-MBP (d) and NF-H (e) antibody showed the decreased expression of the axonal and myelin markers in $N R D c^{+/-}$brain. The sections were double-stained with fluorescent Nissl. Scale bars, c,d,e; $500 \mu \mathrm{m}$. (f) Immunoblot analysis of whole brain extracts using anti-NRDc, NF-H, MBP antibodies revealed the reduced expression of those proteins in $N R D c^{+/-}$brains compared with their wild-type littermates. Full-length blots are presented in Supplementary Figure 9. (g) Electron micrographs of $N R D c^{+/+}$(left) and $N R D c^{+/-}$(right) corpus callosum at P120. Scale bars, $2 \mu \mathrm{m}$. (h) Scatter plots of g-ratio against axon diameters of $N R D c^{+/+}$(red) and $N R D c^{+/-}$ 
(black) corpus callosum at P120.

Figure 6 Transgenic overexpression of neuronal NRDc induces hypermyelination in the CNS. (a) Immunoblot analysis of total brain extracts from wild-type littermates (Wt) and CamKII-NRDc mice (NRDc-Tg) at P90 with anti-NRDc antibody. (b,c) Immunohistochemistry of sections from NRDc-Tg brains (right) using anti-NRDc antibody showed neuronal overexpression of NRDc in the cortex (b) and hippocampus (c) compared with Wt brains (left). Scale bars, $250 \mu \mathrm{m}$. (d) Brains of Wt and NRDc-Tg mice were divided into two regions at the border of cerebral cortex and cerebellum. Total brain extracts from frontal region (left panel) and posterior region (right panel) were analyzed by immunoblotting using anti-NRDc, CNP, MBP and NF-H antibody. Note that the increased expression of NRDc, CNP and MBP were detected only in the frontal region. Full-length blots are presented in Supplementary Figure 9. (e) Expression of myelin marker, MBP in the corpus callosum was increased in NRDc-Tg (right) compared with Wt (left). Scale bars, $500 \mu \mathrm{m}$. (f) Electron micrographs of corpus callosum from NRDc-Tg (right) and Wt (left) mice at P30. Scale bars, $500 \mathrm{~nm}$. (g,h) Myelin sheaths are thicker in NRDc-Tg mice compared with Wt. Scatter plots of g-ratios against axon diameters at P30 in NRDc-Tg (black) and wild-type (red) corpus callosum $(\mathbf{g})$ and average g-ratio $(\mathbf{h})$ are shown. Data represent the mean \pm s.e.m. for no fewer than 1000 myelinated fibers from each group $(n=4$, for each genotype).

Figure 7 Impaired motor activity and cognitive deficits in $N R D c^{-/-}$mice. Mice at the age of 90 to 180 days were analyzed with a battery of behavioral tests (Age-matched littermates, $\mathrm{n}=12 ; N R D c^{+/+}, \mathrm{n}=6 ; N R D c^{-/-}$). (a) Grip strength test, showing the reduced forelimb grip strength of $N R D c^{-/}$mice. $(\mathbf{b}, \mathbf{c})$ The ability of mice to traverse a narrow beam to reach a dark box was analyzed in a beam test. $N R D c^{-/}$mice moved slower (b) and slipped more frequently (c) than $N R D c^{+/+}$mice. (d) Latency to fall from 
a rotating drum was measured in a rotarod test. $N R D c^{-/}$mice showed a shorter latency. (e) The percentage of correct choices in the T-maze forced alteration task, showing impaired working memory in $N R D c^{-/}$mice. (f) The percentage of correct choices in the T-maze left-right discrimination task, showing no difference between $N R D c^{+/+}$and $N R D c^{-/-}$mice. The genotype effect was analyzed by a two-way repeated ANOVA in all tests.

Figure 8 NRDc regulates NRG1 shedding through BACE1 and TACE. (a) FLAG-tagged NRDc, V5-tagged BACE1, TACE, and HA-tagged NRG1-type1 were co-expressed in COS7 cells. Immunoblot analyses with the indicated antibodies were performed. Note that the cleaved N-terminal fragment (NTF) of NRG1 in the total cell lysates (TCL) is increased by the addition of NRDc to BACE1 (lane 3 and 4), while co-expression of NRDc with TACE clearly increased the NTF in the conditioned medium (CM) (lane 5 and 6). (b) Quantification of the cleaved NTF in the TCL and CM by densitometry. The ratio was arbitrarily set at 1 in control vector-transfected cells. Data represent the mean \pm s.e.m. in five independent experiments. $*, p<0.05$. (c) Immunoprecipitation of the co-transfected cell lysate with an anti-FLAG antibody showed a complex of NRDc, BACE1 and NRG1. Only the full-length (FL) of NRG1 is shown here. (d) Expression of endogenous NRG1-typeI and NRG1-typeIII in MEF cells isolated from $N R D c^{+/+}$or $N R D c^{-/-}$mice. Note that the full-length (FL) of NRG1-typeI is increased and the cleaved C-terminal fragment $(\mathrm{CTF})$ is decreased in $N R D c^{-/-}$cells (lane2), and that the cleaved NTF of NRG1-typeIII is decreased in $N R D c^{-/}$cells (lane4). (e) Immunoblot analysis of brain extracts from control wild-type $(+/+)$ and $N R D c^{-/-}$ $(-/-)$ mice at P14 (n $=3$ in each genotype) with indicated antibodies. Note that the full-length (FL) of NRG1 ( 140 kD) is increased, while NTF of NRG1-typeIII (75 kD) is decreased in $N R D c^{-/-}$brains. The mature form of BACE1 in $N R D c^{+/+}$brain extracts is indicated by an arrow. The ratio of the mature form to immature form is higher in 
$N R D c^{+/+}$brains compared with $N R D c^{-/-}$brains. 


\section{Online Methods}

\section{Generation of mutant mice}

NRDc-deficient

mice

(Acc.No.

CDB0466K:

http://www.cdb.riken.jp/arg/mutant\%20mice\%20list.html) were generated by gene targeting in $\mathrm{TT}^{37}$ embryonic stem (ES) cells as described at http://www.cdb.riken.jp/arg/protocol.html. The targeting vector was designed to replace exon

(Supplementary

Figure

1a)

with

DT-A/lox71/LacZ-pA/frt/PGK-Neo/frt/loxP/pA

cassette

(http://www.cdb.riken.jp/arg/cassette.html), and successful homologous recombination in TT2-ES cells was confirmed by PCR (Forward primer (\#1 in Supplementary Figure 1a): 5'- CCTCAGCTCAGCCACTAGCCCTTG -3', Reverse primer (\#2): 5'TCCAACCCGGCCTCGCTCACACCGA-3') and Southern blotting in 2 clones, which yielded highly chimeric mice, respectively. We intercrossed the heterozygous offspring (F1) to obtain homozygous mutants (F2), which were genotyped with tail DNA by PCR using a mixture of three primers. Forward primer (\#3 in Supplementary Figure 1a): 5'- GGATGTCCAGCGCTACGACT-3', Forward primer (\#4): 5'ATCGATGATATCAGATCCCC-3', Reverse primer (\#5): 5'CCTCCCCTTTACCATCCATC-3'. Primers \#3 and \#5 amplify the wild-type allele (674 bp) and primers \#4 and \#5 amplify the mutated allele (380 bp) (Supplementary Figure 1b). Two strains of NRDc-deficient mice, derived from two different clones of ES cells, showed identical phenotypes. All analysis of homozygous mutant mice were done using F2 generation, which has $75 \%$ of $\mathrm{C} 57 \mathrm{BL} / 6$ and $25 \%$ of CBA background. For the analysis of heterozygous mutant mice, F2 mice were backcrossed with C57BL/6 more than 6 times to exclude the possible effect of genetic heterogeneity on the phenotype of the heterozygous mice.

For the generation of neuron-specific NRDc-overexpressing transgenic mice, PCR-amplified cDNA encoding mouse $N R D c$ was cloned downstream of the CamKII $\alpha$ 
promotor $^{26}$. The construct was excised at a unique PacI site for DNA microinjection of fertilized mouse oocytes. Routine genotyping of tail DNA was conducted by PCR using the following primers. Forward primer: 5'-CCATAGCAACTGCCTCTTTG-3', Reverse primer: 5'- GATCGCTGGGAGACTTGATG-3'.

Mice were housed in environmentally controlled rooms of the Institute of Laboratory Animals, Graduate School of Medicine, Kyoto University, under the Institute's guidelines for animal and recombinant DNA experiments.

\section{Cells, plasmids and transfections}

Mouse embryonic fibroblasts (MEF) were isolated from either $N R D c^{+/+}, N R D c^{+/-}$or $N R D c^{-/-}$embryos at embryonic day 14.5 (E14.5), and maintained in Dulbecco's modified Eagle's medium (DMEM) containing $5 \%$ fetal bovine serum, $100 \mathrm{U} / \mathrm{ml}$ of penicillin, $100 \mathrm{mg} / \mathrm{ml}$ of streptomycin, and $2 \mathrm{mM} \mathrm{l-glutamine.} \mathrm{MEF} \mathrm{were} \mathrm{passed}$ according to the $3 \mathrm{~T} 3$ protocol and immortalized ${ }^{38}$. COS7 cells were cultured in DMEM containing $10 \%$ fetal bovine serum, $100 \mathrm{U} / \mathrm{ml}$ of penicillin, $100 \mathrm{mg} / \mathrm{ml}$ of streptomycin, and $2 \mathrm{mM}$ l-glutamine. Expression plasmid for human NRDc ( $p c D N A 3-h N R D c-F L A G)$ was described previously ${ }^{19}$. Expression plasmids for V5-tagged human BACE1 and HA-tagged human NRG1-type I were generous gifts from A. Kinoshita and A. Sehara, respectively. The transfection of plasmids into COS7 cells was carried out using FuGENE6 (Roche) according to the manufacturer's instructions.

\section{Immunoblot analysis and immunoprecipitation}

The preparation of total cell extract, immunoblotting and immunoprecipitation were performed as described previously ${ }^{19}$. For the preparation of brain extract, whole brain was homogenized by sonication in lysis buffer containing $10 \mathrm{mM}$ Tris- $\mathrm{HCl}$ ( $\mathrm{pH} 7.4)$, $150 \mathrm{mM} \mathrm{NaCl}, 1 \% \mathrm{NP}-40$ and protease inhibitor cocktail (Complete Mini, Roche Diagnostics), followed by ultra-centrifugation at $100,000 \mathrm{~g}$ for $20 \mathrm{~min}$ at $4{ }^{\circ} \mathrm{C}$. 
Primary antibodies used for the immunoblot analysis and immunoprecipitation were as follows; mouse monoclonal antibody for NRDc (\#23) $)^{19}$, anti-glyceraldehyde-3 phosphate dehydrogenase (GAPDH) (Research Diagnostics), anti-neuregulin (NRG-C) (Santa Cruz) for detection of the full-length and the cleaved C-terminus of neuregulin typeI and III, anti-neuregulin typeIII (Chemicon / Millipore) for detection of the full-length and the cleaved N-terminus of neuregulin typeIII, anti-BACE1 (EE-17) (Sigma), anti-PDGFR- $\alpha$ (Santa Cruz), anti-Olig2 (IBL, Japan), anti-GST $\pi$ (Stressgen), anti-MBP (MBL, Japan), anti-CNP (Chemicon), anti-NF-H (Sigma), anti-HA (HA11) (Covance), anti-FLAG M2 (Sigma), and anti-V5 (Invitrogen).

\section{Histological procedures and immunohistochemistry}

Mice were anesthetized and transcardially perfused with $4 \%$ paraformaldehyde (PFA) in PBS. Tissues were post-fixed in $4 \%$ PFA for $16 \mathrm{hr}$ and embedded in paraffin in the traditional way, and sectioned at $6 \mu \mathrm{m}$ thickness on a microtome (Leica, RM2125RT). Standard histological methods including hematoxylin and eosin (H\&E) staining, Klüver-Barrera staining and silver impregnation by the modified Bielschowsky method were performed with deparaffinized sections. For immunohistochemical staining, primary antibodies were used at the following dilutions after antigen retrieval; rabbit polyclonal antibody against NRDc, which was raised in our laboratory ${ }^{39}$, at $8 \mu \mathrm{g} / \mathrm{ml}$; mouse anti-neurofilament-H (NF-H) (Sigma) at 1:200; mouse anti-2', 3'-cyclic nucleotide 3'-phosphodiesterase (CNP) (Chemicon) at 1:200; rat anti- MBP at 1:500, and rabbit anti-Olig2 (IBL) at 1:200. After the incubation with primary antibodies, the sections were washed in PBS and incubated with the secondary antibody, i.e. Alexa Fluor 488 or 594 goat anti-mouse or anti-rabbit IgG (Invitrogen) at 1:200 for $1 \mathrm{hr}$, followed by counterstaining with the fluorescent Nissl staining, Neuro Trace (Invitrogen). The stained sections were examined under the fluorescence microscope, and images were exported as eight-bit TIFF files and processed using ImageJ Version 
1.30 .

\section{Electron Microscopy and Morphometry}

Mice were anesthetized and fixed via transcardial perfusion with $4 \%$ PFA and $2 \%$ glutaraldehyde in $0.1 \mathrm{M}$ phosphate buffer. A block of approximately one $\times$ one $\times$ two $\mathrm{mm}^{3}$ was removed from the body of corpus callosum at the level of the dorsal hippocampus and from the sciatic nerve, incubated for $2 \mathrm{hr}$ at $4{ }^{\circ} \mathrm{C}$ in the same fixative, and contrasted with $1 \%$ osmic acid in PBS. Tissues were dehydrated in an ethanol gradient from $50 \%$ to $100 \%$ and embedded in Epon. Semi-thin sections $(0.9 \mu \mathrm{m})$ were stained with toluidine blue for survey by light microscopy. Ultra-thin sections (80 nm) were cut and stained with $2 \%$ uranyl acetate (Watson's modified method) and Reynolds lead citrate, and analyzed with a HITACHI H-7650 transmission electron microscope. Non-overlapping digitalized images of fiber cross-sections were obtained and analyzed using Image Pro Plus 3.0 software (Media Cybernetics Inc.). The g-ratio was determined by dividing the diameter of an axon (without myelin) by that of the total fiber diameter (axon + myelin sheath) from 300 to 1000 fibers in the corpus callosum (at least 6 animals per genotype).

\section{Behavioral analysis.}

Age-matched littermates of $N R D c^{+/+}(\mathrm{n}=12)$ and $N R D c^{-/}(\mathrm{n}=6)$ mice (P90 to P180) were analyzed with a comprehensive behavioral test battery including grip strength test, wire-hanging test, rotarod test, beam test, hot plate test and T-maze tests. Mice were housed in groups of three to four in clear plastic cages maintained in a room on a $12 \mathrm{hr}$ light/dark schedule with food and water provided ad libitum, unless specified otherwise. All experiments were performed in the light phase of the circadian cycle between 10:00 A.M. and 4:00 P.M. All behavioral testing procedures were approved by the Animal Care and Use Committee of Kyoto University, Kyoto, Japan. 


\section{Grip strength and wire-hanging tests}

Neuromuscular strength was examined by the grip strength and wire-hanging tests. A grip strength meter (O’Hara \& Co., Tokyo) was used to assess forelimb grip strength. Mice were lifted and held by their tail so that their forepaws could grasp a wire grid. The mice were then gently pulled backward by the tail with their posture parallel to the surface of the table until they released the grid. The peak force applied by mouse forelimbs was recorded in Newtons $(\mathrm{N})$. Each mouse was tested three times and the greatest value measured was used for the statistical analysis. In the wire-hanging test, mice were placed on a wire mesh that was then inverted and waved gently, so that the subject gripped the wire. Latency to fall was recorded, with a $60 \mathrm{sec}$ cut-off time.

\section{Rotarod test, beam test and hot plate test}

Motor coordination and balance were assessed with a rotarod test and a beam test. We performed the rotarod test using an accelerating rotarod (UGO Basile Accelerating Rotarod, Italy) by placing a mouse on a rotating drum ( $3 \mathrm{~cm}$ in diameter) and measuring the time each animal was able to maintain its balance on the rod as latency time to fall (seconds). The speed of the rotarod was accelerated from 4 to $40 \mathrm{rpm}$ over a 5 -min period. A beam test was performed to measure the ability of mice to traverse a narrow beam to reach a dark box ${ }^{29}$. The beam, with a rough painted surface, consisted of iron (100 cm long; $2.8 \mathrm{~cm}$ in diameter) placed horizontally $50 \mathrm{~cm}$ above the bench surface. One session of five trials was performed using the beam. Mice were allowed up to 60 sec to traverse each beam. The number of sideslips was recorded for each trial by the Image OF program. The hot plate test was used to evaluate nociception or sensitivity to a painful stimulus. Mice were placed on a hot plate at $55.0 \pm 0.3{ }^{\circ} \mathrm{C}$ (Columbus Instruments, Columbus, Ohio), and latency to the first hind-paw response was recorded. The hind-paw response was either a foot shake or a paw lick.

\section{T-maze forced alternation task}

The forced alternation task was conducted using an automatic T-maze that we devised ${ }^{30}$ 
(O’Hara \& Co., Tokyo, Japan). It was constructed of white plastics runways with walls $25-\mathrm{cm}$ high. The maze was partitioned off into 6 areas by sliding doors that can be opened downward. The details of protocols were described previously ${ }^{30}$. After the adaptation session, mice were subjected to a forced alternation protocol for 7 days (one session consisting of 10 trials per day; cut-off time, $50 \mathrm{~min}$ ). Data acquisition, control of sliding doors, and data analysis were performed by Image TM software (see 'Image analysis').

\section{T-maze Left-right discrimination task}

The left-right discrimination task was conducted using an automatic T-maze and deprivation before the trials as mentioned above and previously ${ }^{30}$. On the day after the adaptation session, mice were subjected to a left-right discrimination task for 20 days (one session consisting of 10 trials, 2 sessions per day; cut-off time, $50 \mathrm{~min}$ ). The mouse was able to freely choose either the right or left arm of the T (A1 and A2). The correct arm was assigned to each mouse randomly. If it chose the correct arm, the mouse received a reward at the end of the arm. Choosing the incorrect arm resulted in no reward and confinement to the arm for $10 \mathrm{sec}$. After the mouse consumed the pellet or the mouse stayed more than $10 \mathrm{sec}$ without consuming the pellet, the door that separated the arm (A1 or A2) and connecting passage-way (P1 or P2) was opened and the mouse could return to the starting compartment (S1), via the connecting passage-way. On the 11th day, the correct arm was changed for reversal learning. A variety of fixed extra-maze clues surrounded the apparatus.

\section{Image analysis}

The applications used for the behavioral studies were based on the public domain NIH Image program (developed at the U.S. National Institutes of Health and available on the Internet at http://rsb.info.nih.gov/nih-image/) and ImageJ program http://rsb.info.nih.gov/ij/, which were modified for each test by Miyakawa (available through O'Hara \& Co., Tokyo, Japan). 


\section{RNA analysis}

Total brain RNA was isolated from snap-frozen brains (at least three mice per genotype, at P5) using Trizol (GIBCO-BRL) according to the manufacturer's protocol. For real-time PCR, first strand cDNA was synthesized from total RNA using random primers and SuperScriptIII reverse transcriptase (Invitrogen). Quantitative real-time PCR was carried out using the ABI Prism 7700 Sequence Detection System and SYBR Green Master Mix following the manufacturer's directions (Applied Biosystems). The results were standardized for comparison by measuring levels of $\beta$-actin mRNA in each sample. Values are expressed as mean \pm s.e.m.

Mouse-specific primers used for real-time PCR were as follows;

NRG1-typeI, 5' primer: CTGTGTCTGCCTGGAA

NRG1-typeI, 3' primer: CAGCCGTTGGATCCAGA

NRG1-typeIII, 5' primer: GCTCTACCAGAAGAGGGTA

NRG1-typeIII, 3' primer: GATTCACCAGTTGCACA

BACE1, 5' primer: ACCATCCTTCCTCAGCAA

$B A C E 1,3$ ' primer: GGGAATGTTGTAGCCACA

$\beta$-actin, 5' primer: CTGACTGACTACCTCATGAAGATCCT

$\beta$-actin, 3' primer: CTTAATGTCACGCACGATTTCC

\section{Statistical analysis}

Data are presented as mean \pm s.e.m. Statistical analyses (Student's two-tailed $t$-test, One-way ANOVA, repeated measures ANOVA) were performed using the STATVIEW Software package (SAS institute).

37. Yagi, T. et al. A novel ES cell line, TT2, with high germline-differentiating potency. Anal Biochem 214, 70-6 (1993).

38. Todaro, G. J. \& Green, H. Quantitative studies of the growth of mouse embryo cells 
in culture and their development into established lines. J Cell Biol 17, 299-313 (1963).

39. Hospital, V. et al. The metalloendopeptidase nardilysin (NRDc) is potently inhibited by heparin-binding epidermal growth factor-like growth factor (HB-EGF). Biochem J 367, 229-38 (2002). 
1. Nave, K. A. \& Trapp, B. D. Axon-glial signaling and the glial support of axon function. Annu Rev Neurosci 31, 535-61 (2008).

2. Simons, M. \& Trotter, J. Wrapping it up: the cell biology of myelination. Curr Opin Neurobiol 17, 533-40 (2007).

3. Hartline, D. K. \& Colman, D. R. Rapid conduction and the evolution of giant axons and myelinated fibers. Curr Biol 17, R29-35 (2007).

4. McTigue, D. M. \& Tripathi, R. B. The life, death, and replacement of oligodendrocytes in the adult CNS. $J$ Neurochem 107, 1-19 (2008).

5. Griffiths, I. et al. Axonal swellings and degeneration in mice lacking the major proteolipid of myelin. Science 280, 1610-3 (1998).

6. Lappe-Siefke, C. et al. Disruption of Cnp1 uncouples oligodendroglial functions in axonal support and myelination. Nat Genet 33, 366-74 (2003).

7. Michailov, G. V. et al. Axonal neuregulin-1 regulates myelin sheath thickness. Science 304, 700-3 (2004).

8. Nave, K. A. \& Salzer, J. L. Axonal regulation of myelination by neuregulin 1. Curr Opin Neurobiol 16, 492-500 (2006).

9. Mei, L. \& Xiong, W. C. Neuregulin 1 in neural development, synaptic plasticity and schizophrenia. Nat Rev Neurosci 9, 437-52 (2008).

10. Falls, D. L. Neuregulins: functions, forms, and signaling strategies. Exp Cell Res 284, 14-30 (2003).

11. Montero, J. C., Yuste, L., Diaz-Rodriguez, E., Esparis-Ogando, A. \& Pandiella, A. Differential shedding of transmembrane neuregulin isoforms by the tumor necrosis factor-alpha-converting enzyme. Mol Cell Neurosci 16, 631-48 (2000).

12. Shirakabe, K., Wakatsuki, S., Kurisaki, T. \& Fujisawa-Sehara, A. Roles of Meltrin beta /ADAM19 in the processing of neuregulin. J Biol Chem 276, 9352-8 (2001).

13. Willem, M. et al. Control of peripheral nerve myelination by the beta-secretase BACE1. Science 314, 664-6 (2006).

14. $\mathrm{Hu}, \mathrm{X}$. et al. Bace1 modulates myelination in the central and peripheral nervous system. Nat Neurosci 9, 1520-5 (2006).

15. $\mathrm{Hu}, \mathrm{X}$. et al. Genetic deletion of BACE1 in mice affects remyelination of sciatic nerves. Faseb J 22, 2970-80 (2008).

16. Pierotti, A. R. et al. N-arginine dibasic convertase, a metalloendopeptidase as a prototype of a class of processing enzymes. Proc Natl Acad Sci U S A 91, 6078-82 (1994).

17. Chesneau, V. et al. N-arginine dibasic convertase (NRD convertase): a newcomer to the family of processing endopeptidases. An overview. Biochimie 76, 234-40 (1994).

18. Nishi, E., Prat, A., Hospital, V., Elenius, K. \& Klagsbrun, M. N-arginine dibasic convertase is a specific receptor for heparin-binding EGF-like growth factor that 
mediates cell migration. Embo J 20, 3342-50 (2001).

19. Nishi, E., Hiraoka, Y., Yoshida, K., Okawa, K. \& Kita, T. Nardilysin enhances ectodomain shedding of heparin-binding epidermal growth factor-like growth factor through activation of tumor necrosis factor-alpha-converting enzyme. $J$ Biol Chem 281, 31164-72 (2006).

20. Hiraoka, Y. et al. Enhancement of alpha-secretase cleavage of amyloid precursor protein by a metalloendopeptidase nardilysin. J Neurochem 102, 1595-605 (2007).

21. Hiraoka, Y. et al. Ectodomain shedding of TNF-alpha is enhanced by nardilysin via activation of ADAM proteases. Biochem Biophys Res Commun 370, 154-8 (2008).

22. Bernstein, H. G. et al. Histochemical evidence for wide expression of the metalloendopeptidase nardilysin in human brain neurons. Neuroscience 146, 1513-23 (2007).

23. Lu, Q. R. et al. Common developmental requirement for Olig function indicates a motor neuron/oligodendrocyte connection. Cell 109, 75-86 (2002).

24. Fields, R. D. \& Ellisman, M. H. Axons regenerated through silicone tube splices. II. Functional morphology. Exp Neurol 92, 61-74 (1986).

25. Taveggia, C. et al. Neuregulin-1 type III determines the ensheathment fate of axons. Neuron 47, 681-94 (2005).

26. Mayford, M., Wang, J., Kandel, E. R. \& O'Dell, T. J. CaMKII regulates the frequency-response function of hippocampal synapses for the production of both LTD and LTP. Cell 81, 891-904 (1995).

27. Dragatsis, I. \& Zeitlin, S. CaMKIIalpha-Cre transgene expression and recombination patterns in the mouse brain. Genesis 26, 133-5 (2000).

28. Yamasaki, N. et al. Alpha-CaMKII deficiency causes immature dentate gyrus, a novel candidate endophenotype of psychiatric disorders. Mol Brain 1, 6 (2008).

29. Carter, R. J. et al. Characterization of progressive motor deficits in mice transgenic for the human Huntington's disease mutation. J Neurosci 19, 3248-57 (1999).

30. Takao, K. et al. Impaired long-term memory retention and working memory in sdy mutant mice with a deletion in Dtnbp1, a susceptibility gene for schizophrenia. Mol Brain 1, 11 (2008).

31. Thinakaran, G. \& Koo, E. H. Amyloid precursor protein trafficking, processing, and function. J Biol Chem 283, 29615-9 (2008).

32. Calaora, V. et al. Neuregulin signaling regulates neural precursor growth and the generation of oligodendrocytes in vitro. J Neurosci 21, 4740-51 (2001).

33. Wakatsuki, S., Yumoto, N., Komatsu, K., Araki, T. \& Sehara-Fujisawa, A. Roles of meltrin-beta/ADAM19 in progression of Schwann cell differentiation and myelination during sciatic nerve regeneration. J Biol Chem 284, 2957-66 (2009).

34. Taveggia, C. et al. Type III neuregulin-1 promotes oligodendrocyte myelination. Glia 56, 284-93 (2008).

35. Brinkmann, B. G. et al. Neuregulin-1/ErbB signaling serves distinct functions in 
myelination of the peripheral and central nervous system. Neuron 59, 581-95 (2008).

36. Trapp, B. D. \& Nave, K. A. Multiple sclerosis: an immune or neurodegenerative disorder? Annu Rev Neurosci 31, 247-69 (2008). 


\section{Supplementary Information Titles}

Please list each supplementary item and its title or caption, in the order shown below. Please include this form at the end of the Word document of your manuscript or submit it as a separate file.

Note that we do NOT copy edit or otherwise change supplementary information, and minor (nonfactual) errors in these documents cannot be corrected after publication. Please submit document(s) exactly as you want them to appear, with all text, images, legends and references in the desired order, and check carefully for errors.

Journal: Nature Neuroscience

\begin{tabular}{|l|r|}
\hline Article Title: & $\begin{array}{r}\text { Nardilysin Regulates Axonal Maturation and Myelination in the } \\
\text { Central and Peripheral Nervous System }\end{array}$ \\
\hline $\begin{array}{l}\text { Corresponding } \\
\text { Author: }\end{array}$ & Eiichiro Nishi \\
\hline
\end{tabular}

\begin{tabular}{|l|l|}
\hline $\begin{array}{l}\text { Supplementary Item \& } \\
\text { Number }\end{array}$ & \multicolumn{1}{c|}{ Title or Caption } \\
\hline Supplementary Figure 1 & Targeted disruption of the NRDc gene \\
\hline Supplementary Figure 2 & $\begin{array}{l}\text { H \& E stained sections of brain hemisphere of NRDc-/- mice } \\
\text { at P1, P14 and P30 }\end{array}$ \\
\hline Supplementary Figure 3 & Expression of NRDc mRNA (nrd1) in the nervous system \\
\hline Supplementary Figure 4 & $\begin{array}{l}\text { Correlation between the expression level of NRDc and the } \\
\text { level of myelination defect in NRDc-/- mice }\end{array}$ \\
\hline Supplementary Figure 5 & $\begin{array}{l}\text { No difference in the number of OPC between NRDc+/+ and } \\
\text { NRDc-/- brains }\end{array}$ \\
\hline Supplementary Figure 6 & $\begin{array}{l}\text { Delay in the initiation of myelination and hypomyelination in } \\
\text { NRDc-/- mice }\end{array}$ \\
\hline Supplementary Figure 7 & Behavioral tests (Supplement for Figure 7) \\
\hline Supplementary Figure 8 & BACE1 and NRG1 mRNA expression in brain \\
\hline Supplementary Figure 9 & Full-length blots for Figure 1a, 2g, 5f and 6e \\
\hline
\end{tabular}


a

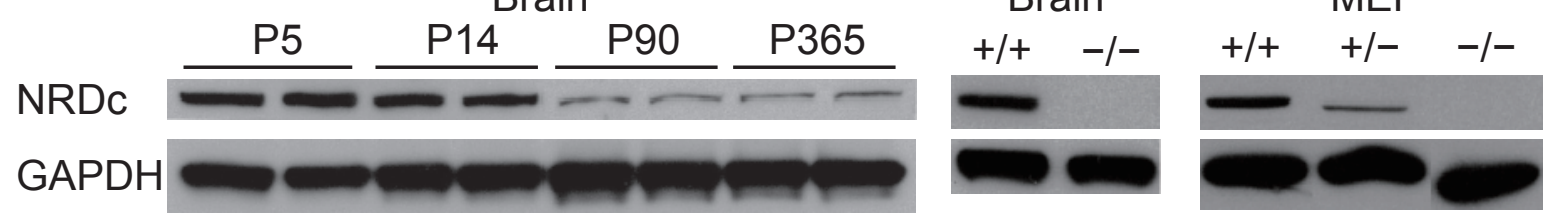

b
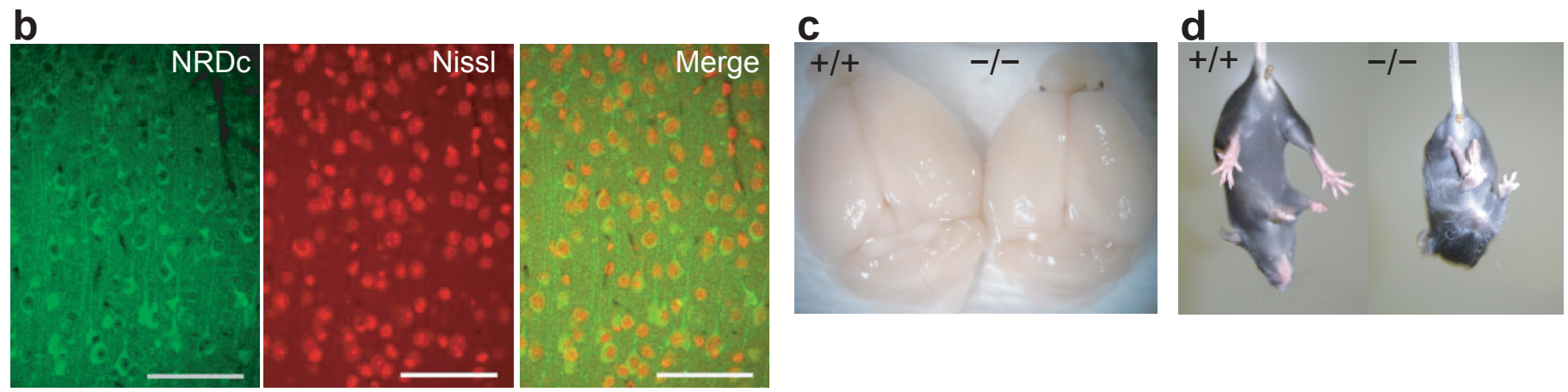

e

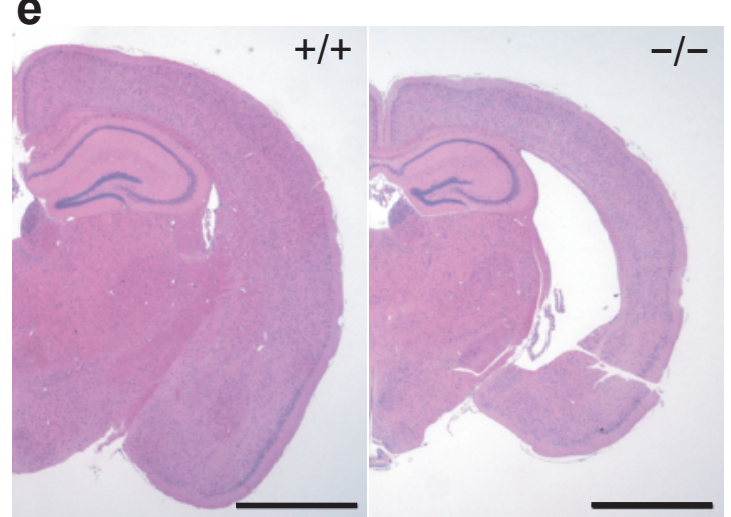

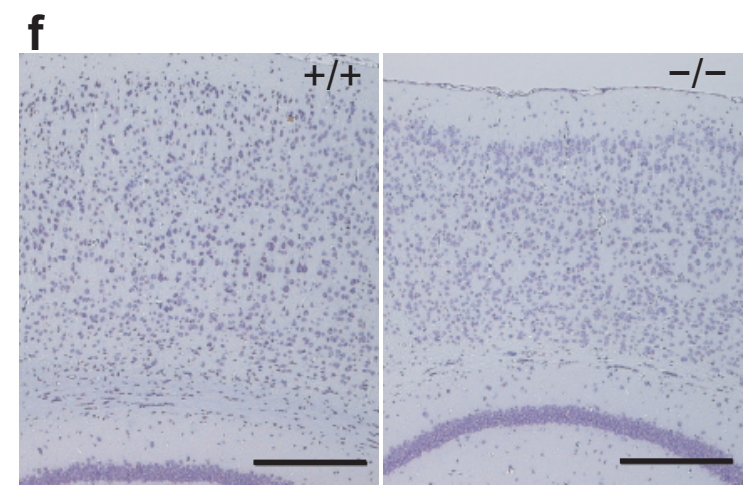

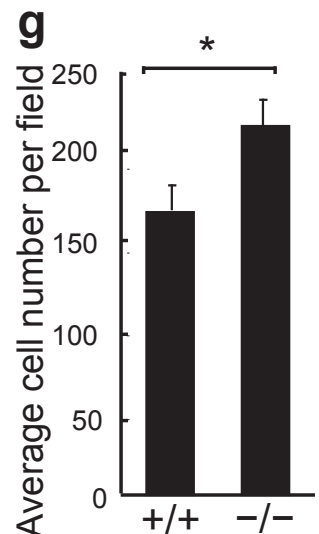


(18. 京都大学

a

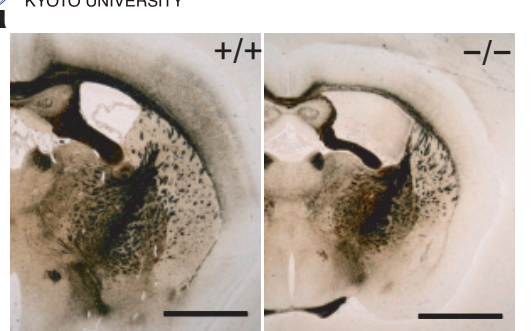

C
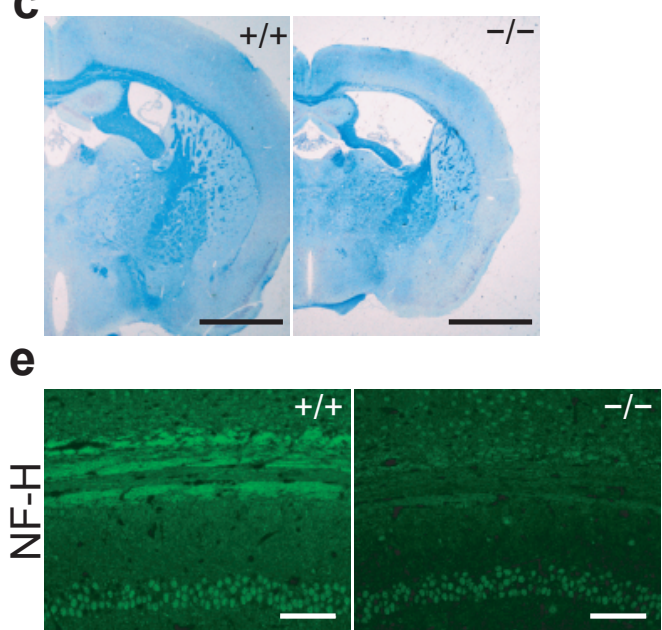

g b

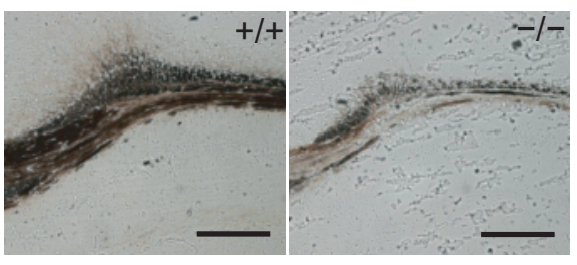

d

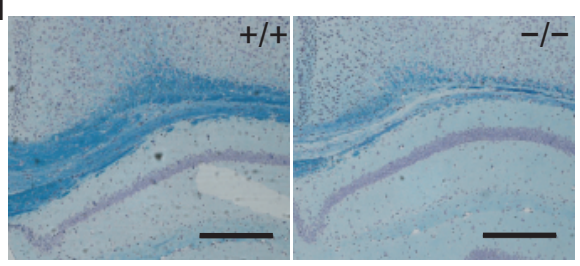

f

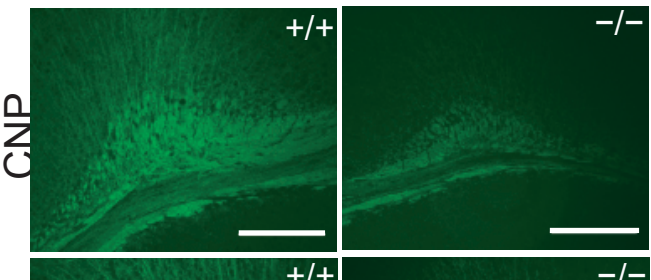

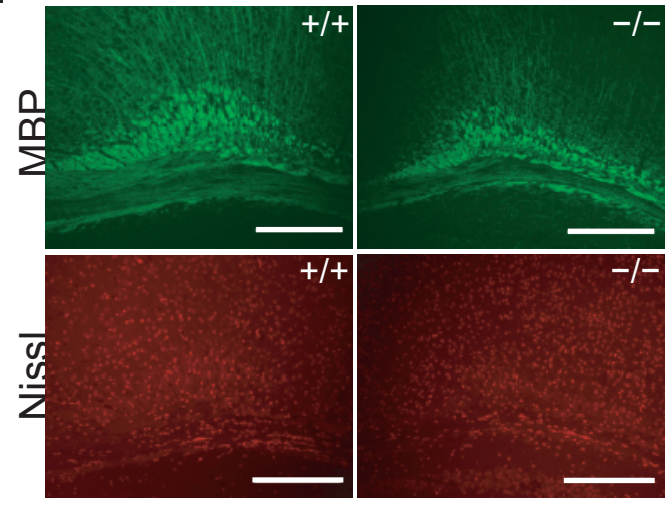

GST- $\pi$

GAPDH

Figure-2 (Nishi) 
京都大学

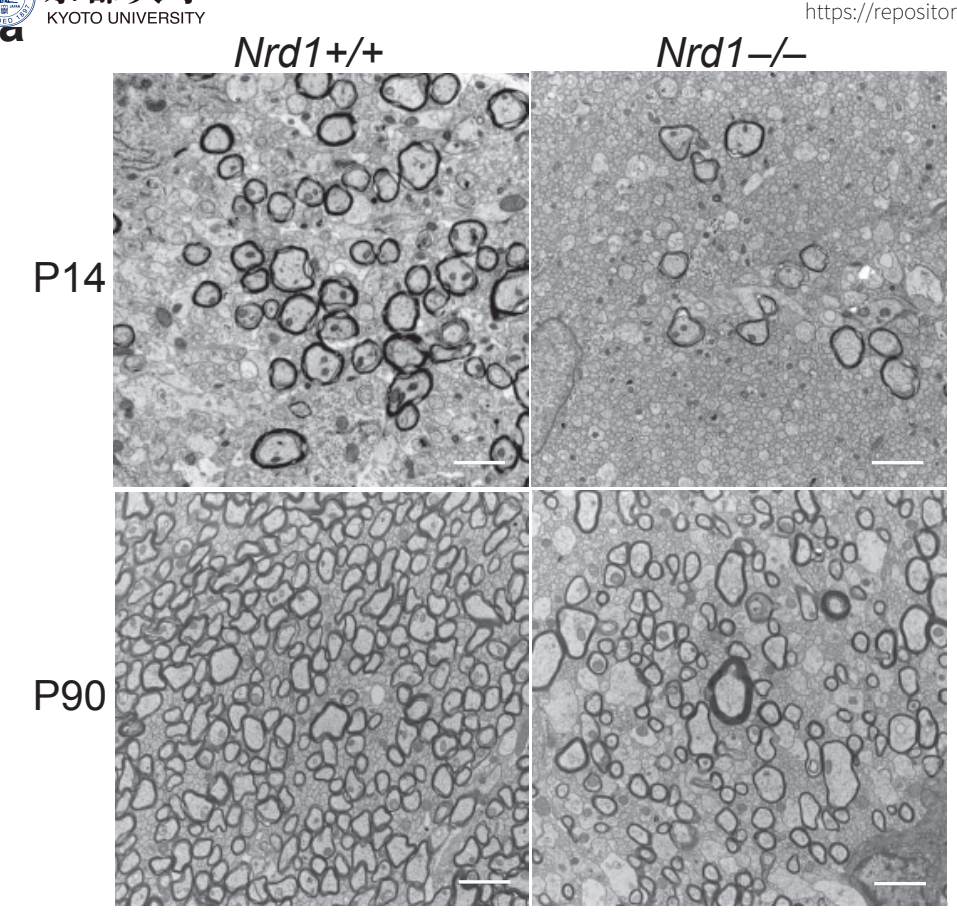

b

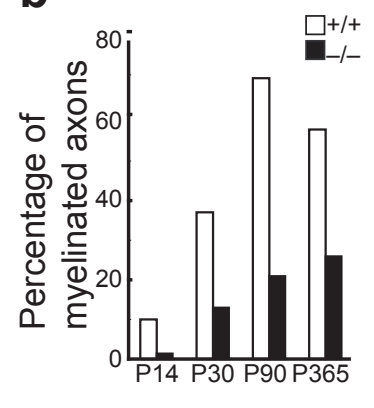

d

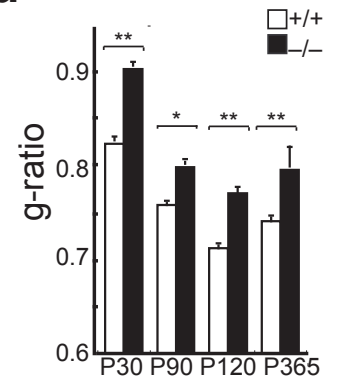

C

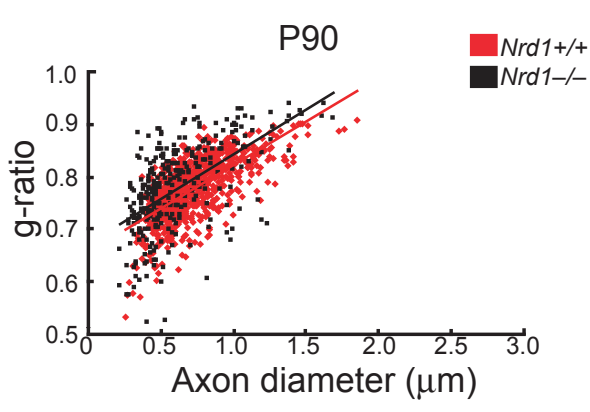

e

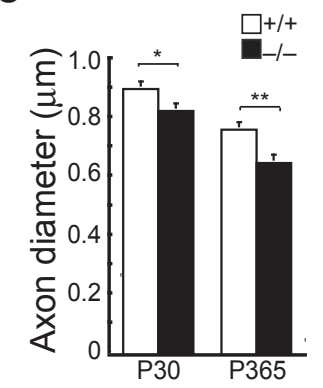


(3) 京都大学

A Self-archived copy in
Kyoto University Research Information Repository

https://reposi by

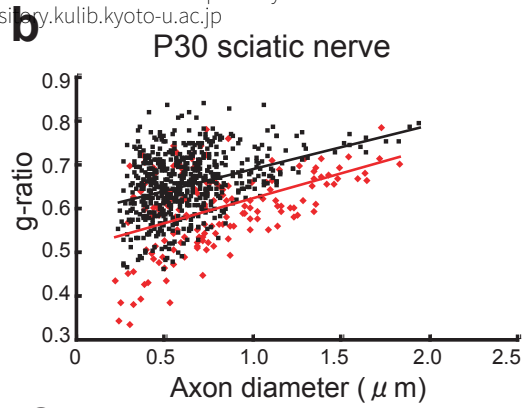

Nrd1-/-

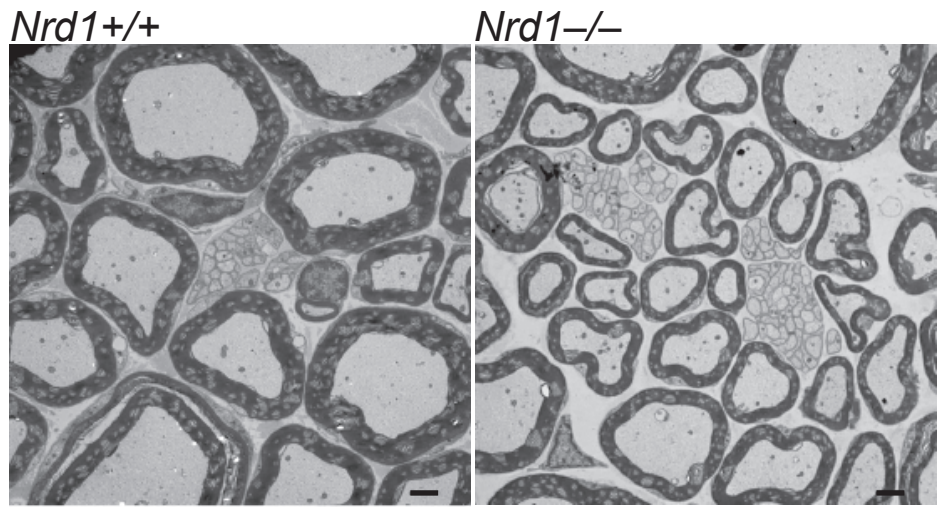

d

Nrd1+/+

Nrd1-/-

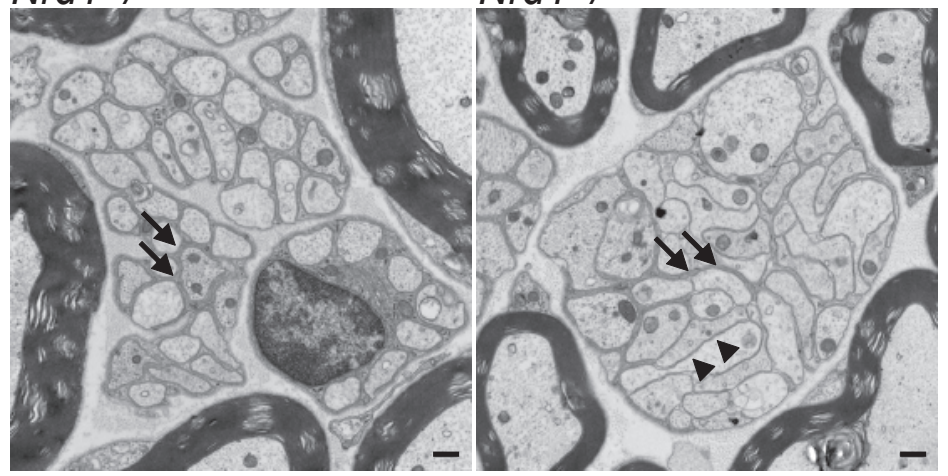

C

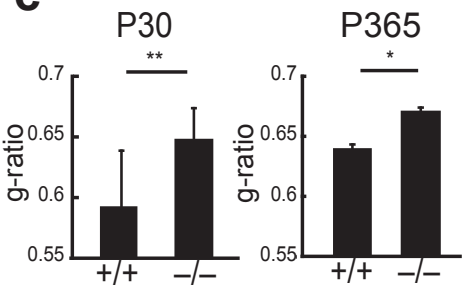

e

Number of axons / remak bundle

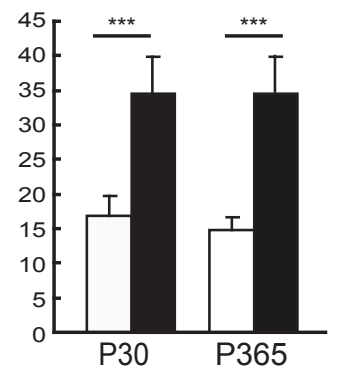

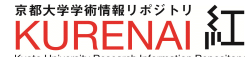

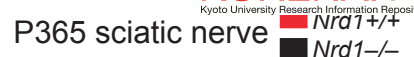
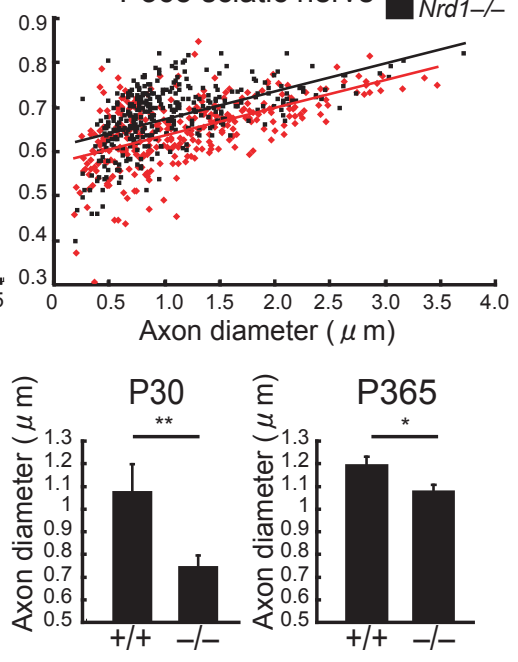

f

Ratio of segregated axon

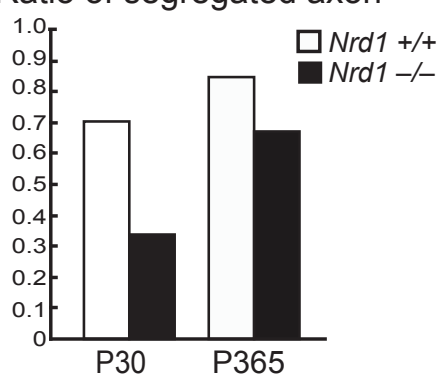


2) 京都大学

IVINUUN KYIVESITY

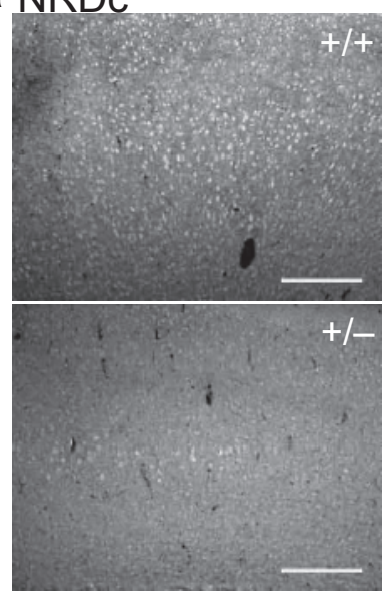

b

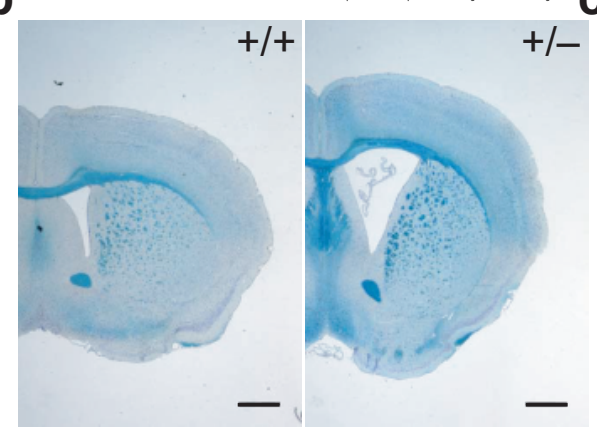

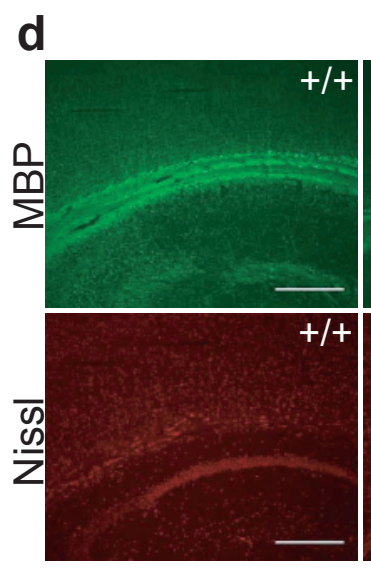

g

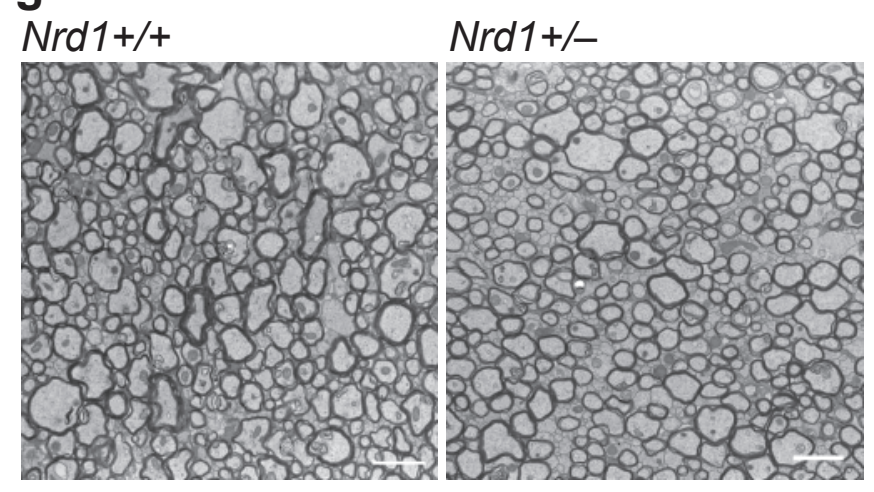

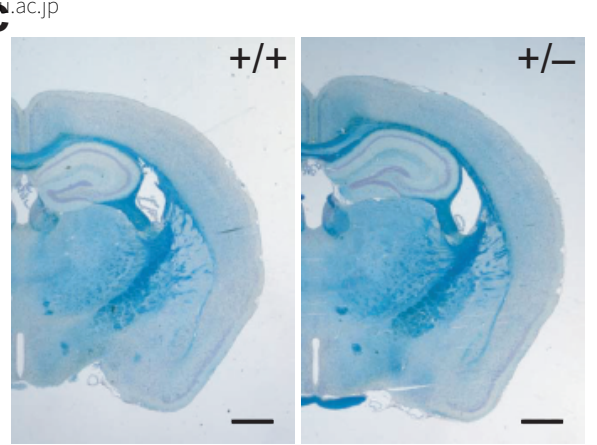

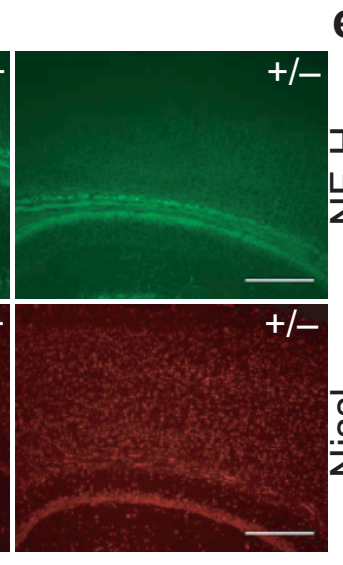

.

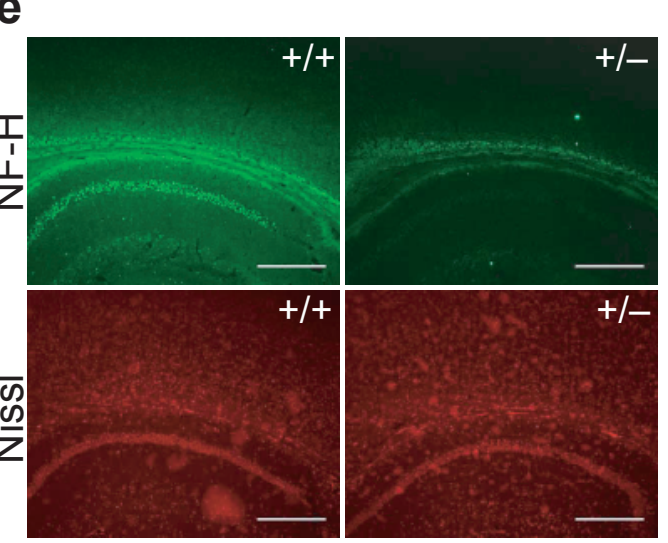

h f

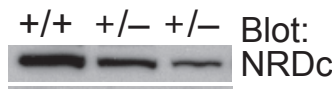

$\longrightarrow$ NF-H

$-3=2.2 \mathrm{MBP}$

$=$ GAPDH

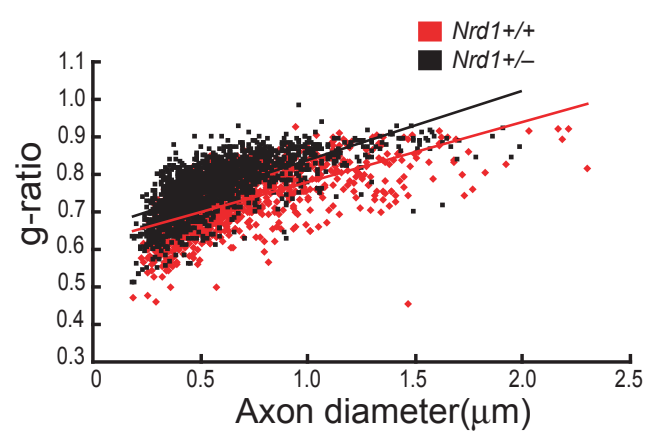


京都大学

a KYOTO UNIVERSITY

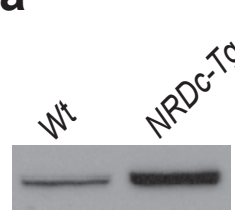

Blot: NRDc
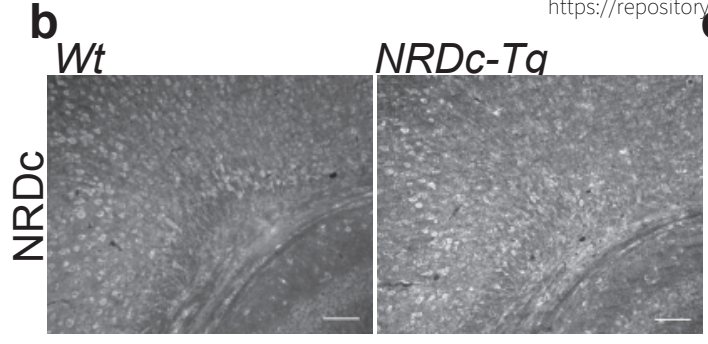

c

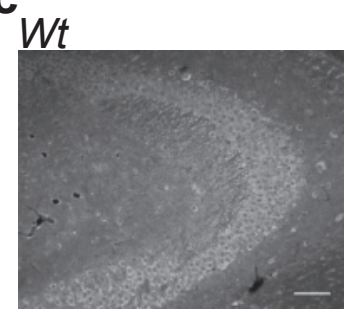

$N R D c-T a$

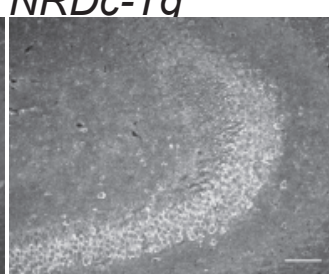

d

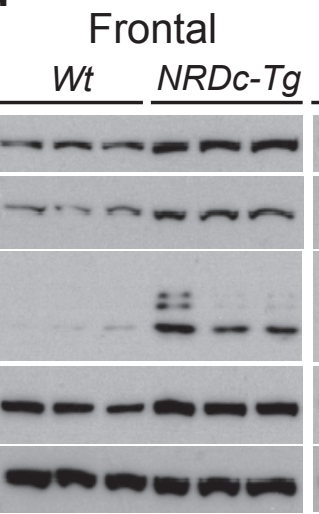

Posterior

Wt NRDc-Tg Blot:

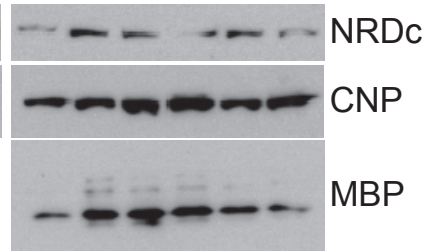

e

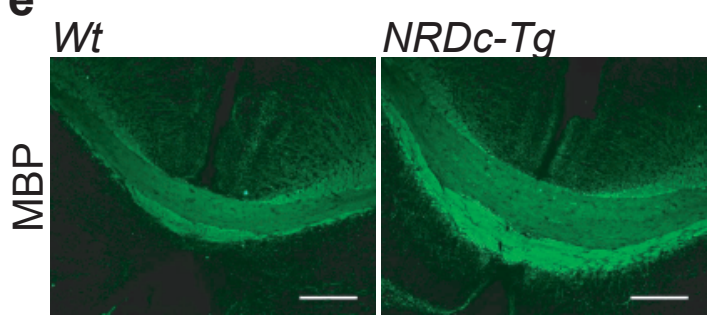

-

GAPDH

f

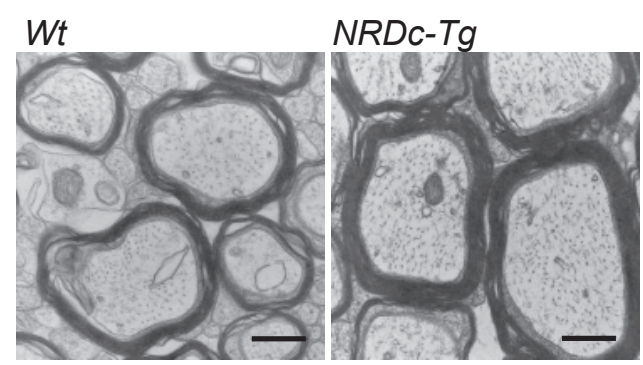

g

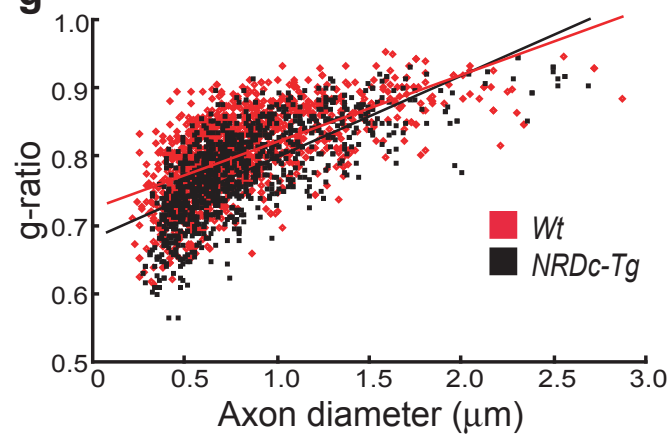

h

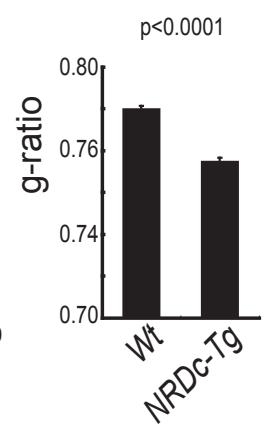


京都大学

KYOTO UNIVERSITY

Grip strength b
Beam test

C Beam test number of slips
Rotarod latency to fall
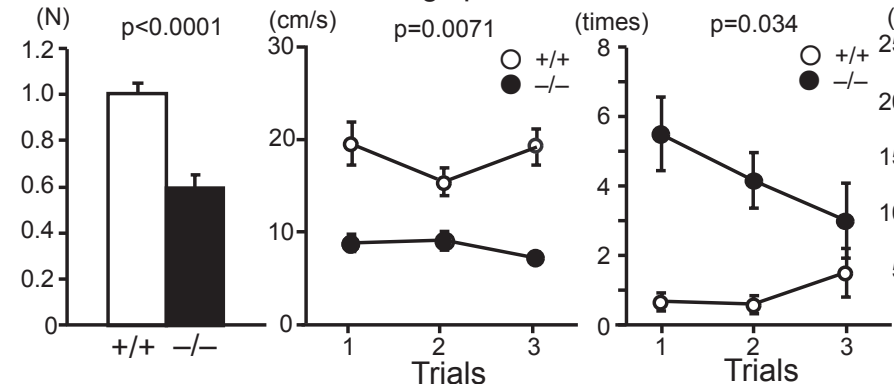

(s) $\quad \mathrm{p}=0.0102$

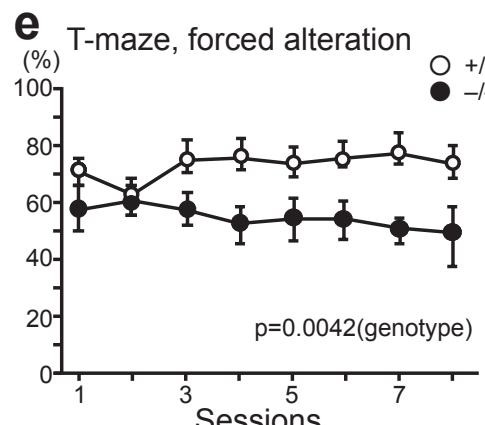

f T-maze, L-R discrimination

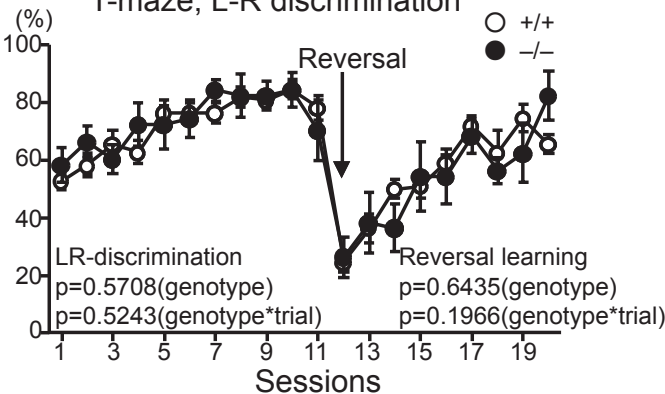


A Self-archived copy in
Kyoto University Research Information Repository

a

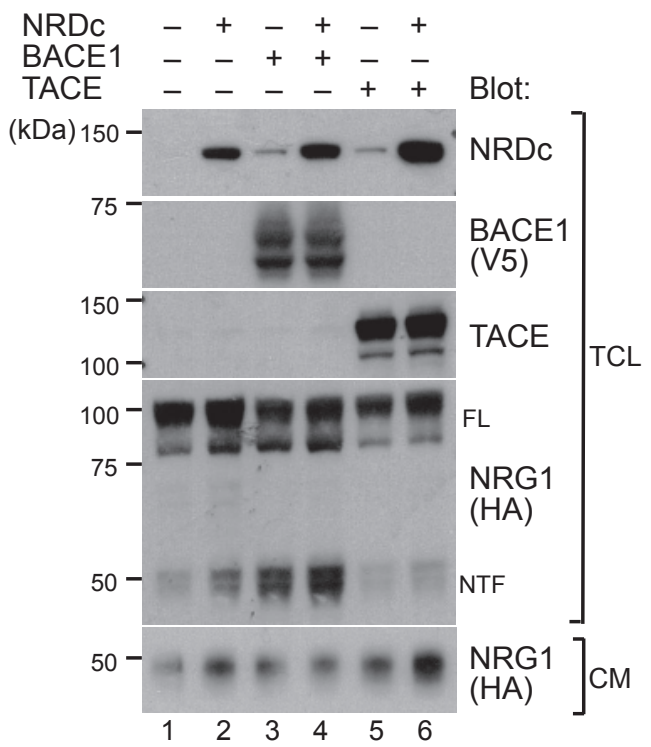

d

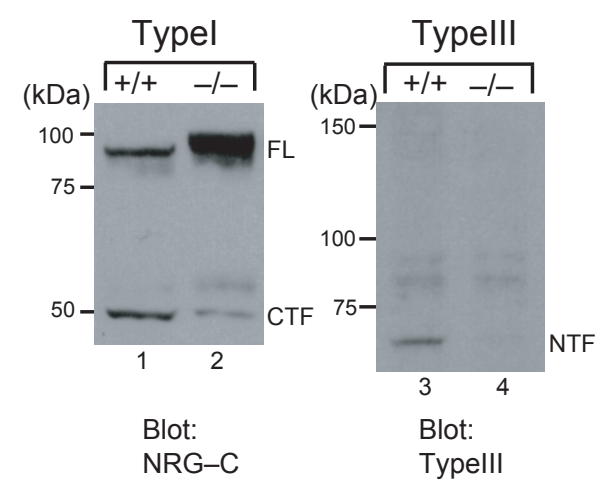

C TCL (HA)

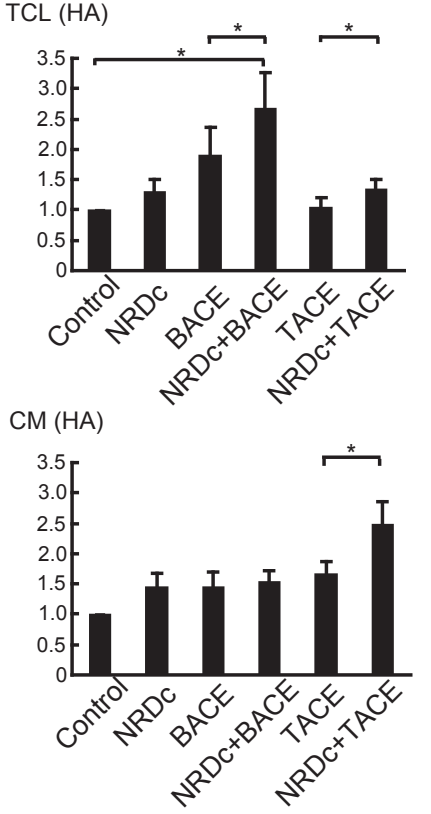

$\begin{array}{llllll}\text { NRDc } & - & + & - & + & \\ \text { BACE1 } & - & - & + & + & \\ \text { NRG1 } & + & + & + & + & \text { Blot: }\end{array}$
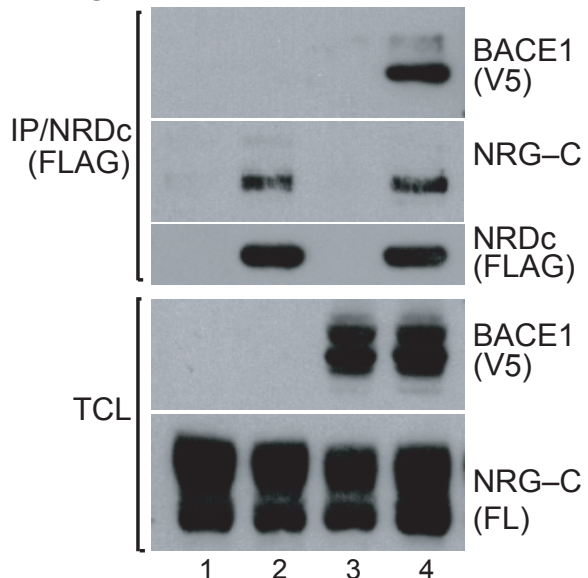

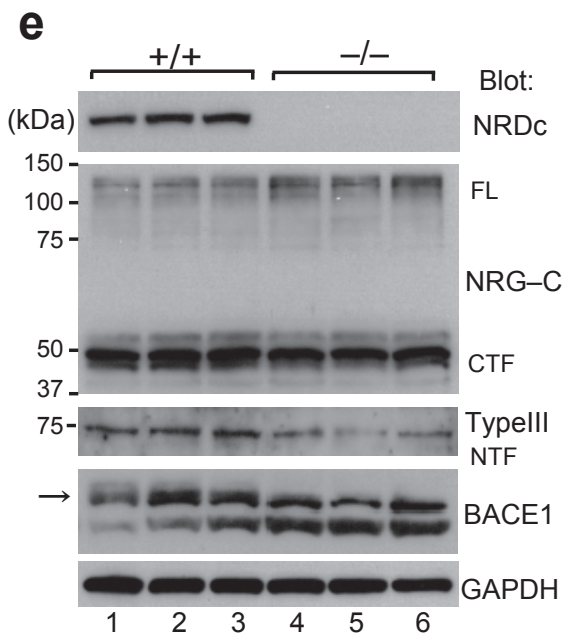

Figure-8 (Nishi) 


\section{Nardilysin Regulates Axonal Maturation and Myelination in the Central and Peripheral Nervous System}

Mikiko Ohno, Yoshinori Hiraoka, Tatsuhiko Matsuoka, Hidekazu Tomimoto, Keizo Takao, Tsuyoshi Miyakawa, Naoko Oshima, Hiroshi Kiyonari, Takeshi Kimura, Toru Kita, and Eiichiro Nishi*

a

Nrd1 in mouse brain

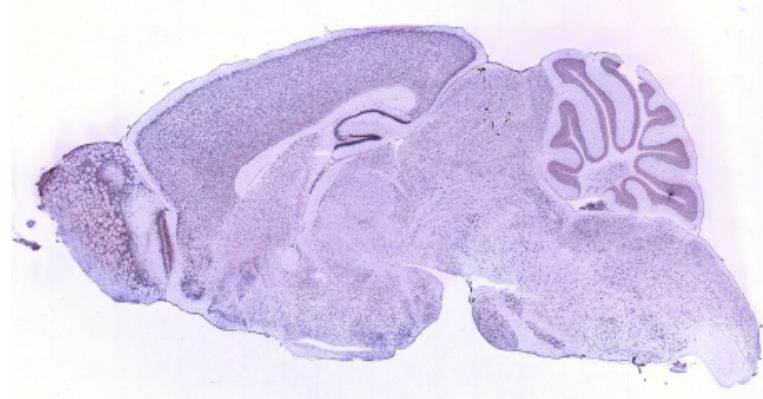

b

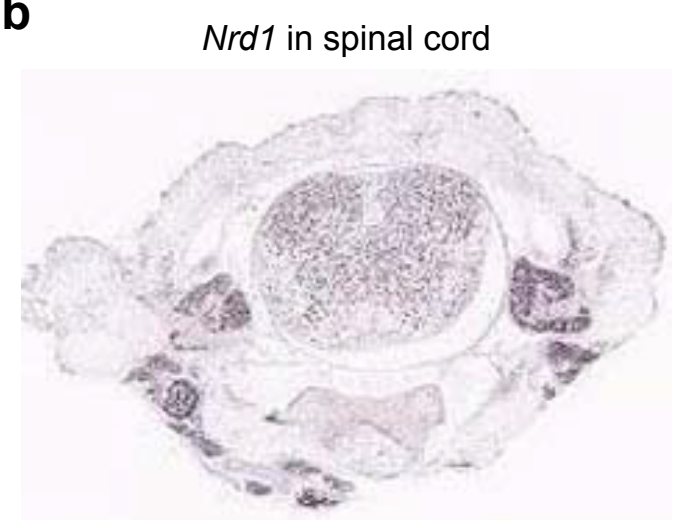

Mbp in mouse brain

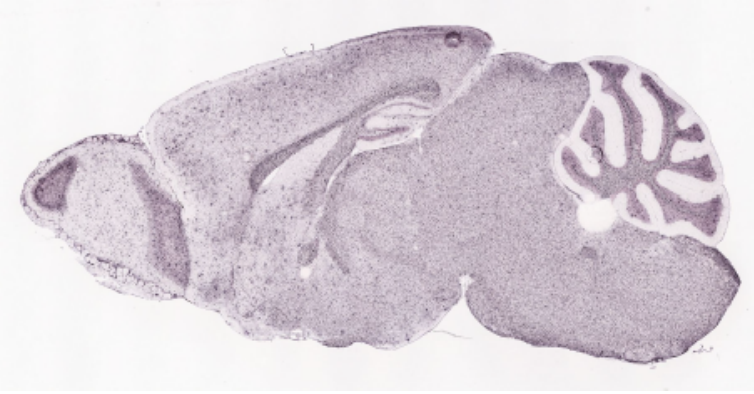

Allen Brain Atlas

\section{Supplementary Figure 1}

Expression of NRDc mRNA (nrd1) in the nervous system.

a, Images of in situ hybridization of $n r d 1$ and $m b p$ in mouse brain, downloaded from the Allen Brain Atlas available from: http://www.brain-map.org.

Note that almost no signal in the corpus callosum is detected for $n r d 1$, while mbp mRNA is strongly expressed in the region.

b, Images of in situ hybridization of $n r d 1$ in mouse spinal cord at the level of C3. Nrd1 expression is detected in the gray matter in spinal cord and dorsal root ganglia. 


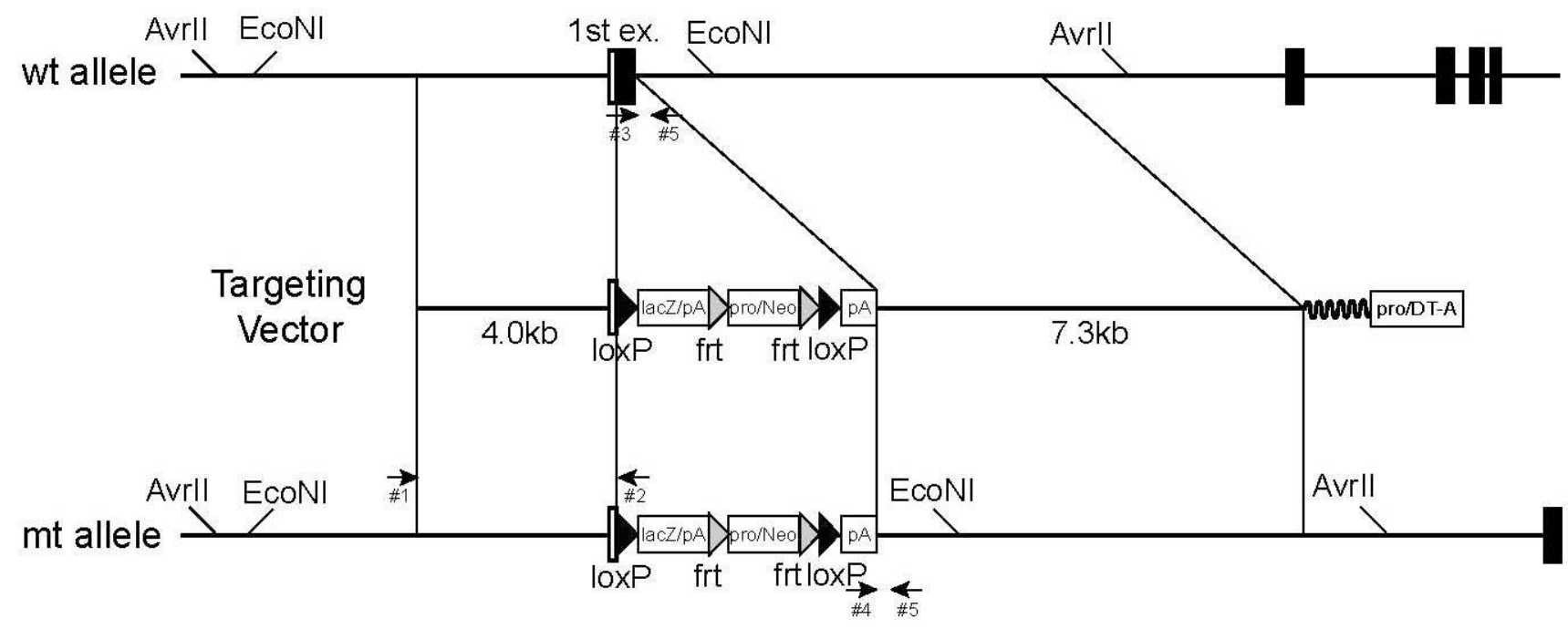

b

$\mathrm{Nrd1}+/+\mathrm{Nrd1}+/-\mathrm{Nrd1}-/-$

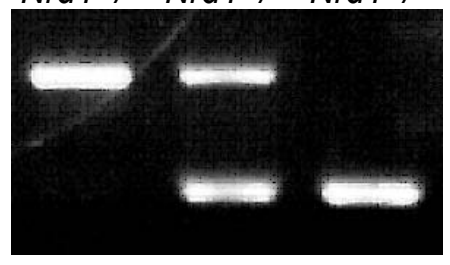

C

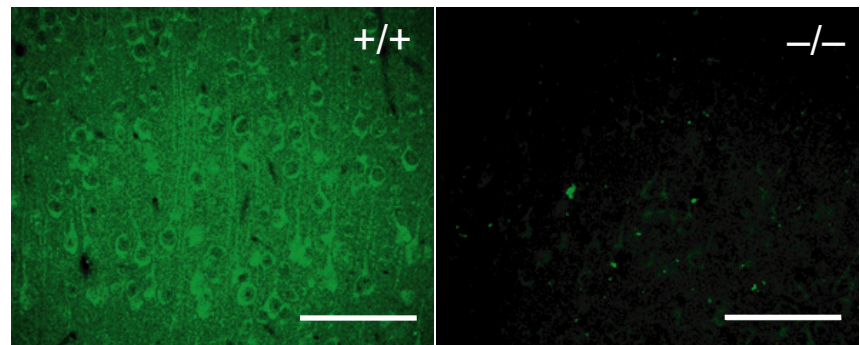

\section{Supplementary Figure 2}

Targeted disruption of the Nrd1 gene.

a, Structure of a part of $n r d 1$, a gene that encodes NRDc (top), the targeting vector (middle),

and the mutated allele (bottom). The targeting vector was designed to replace exon 1. \#1-2; primers used for screening of targeted ES cells. \#3-5; primers used for routine genotyping of the mutant mice. b, PCR genotyping of the NRDc-deficient mice. Primers \#3 and \#5 amplify the wild-type allele (674 bp), while primers \#4 and \#5 amplify the mutant allele (370 bp).

c, Immunohistochemistry using an anti-NRDc antibody with cerebral cortex sections from control wild-type mice (+/+; left) and Nrd1-/- mice (-/-; right) at P30. Scale bars, $250 \mu \mathrm{m}$. 
P1
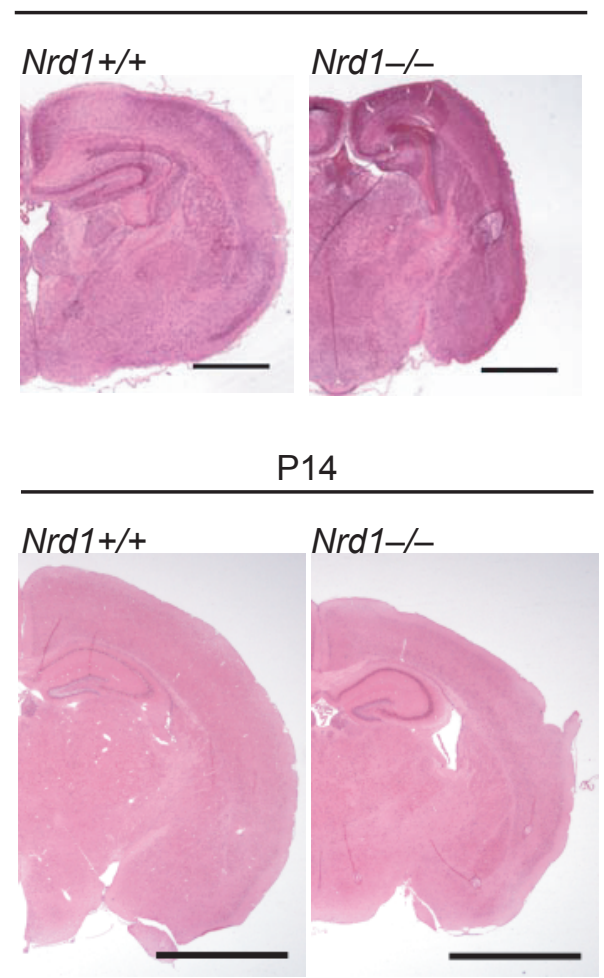

P30

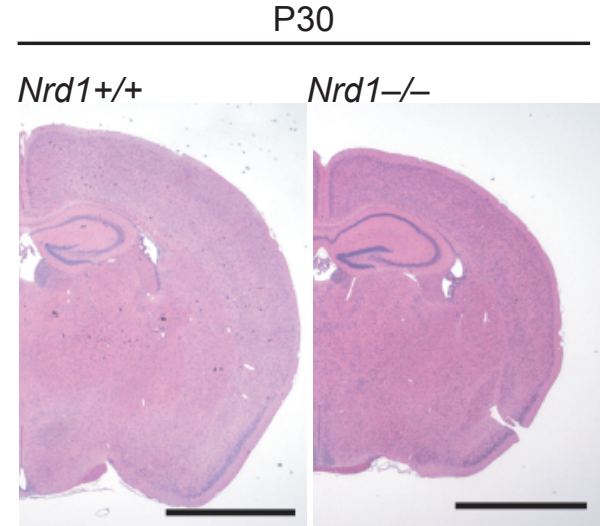

\section{Supplementary Figure 3}

$\mathrm{H} \& \mathrm{E}$ stained sections of brain hemisphere of $\mathrm{Nrd1} 1-/$ mice (right) and wild-type littermates (left) at P1, P14, P30.

The gross anatomy of the Nrd1-/- brains showed no abnormality at these stages. Scale bars, $2 \mathrm{~mm}$. 
京都大学

KYOTO UNIVERSITY

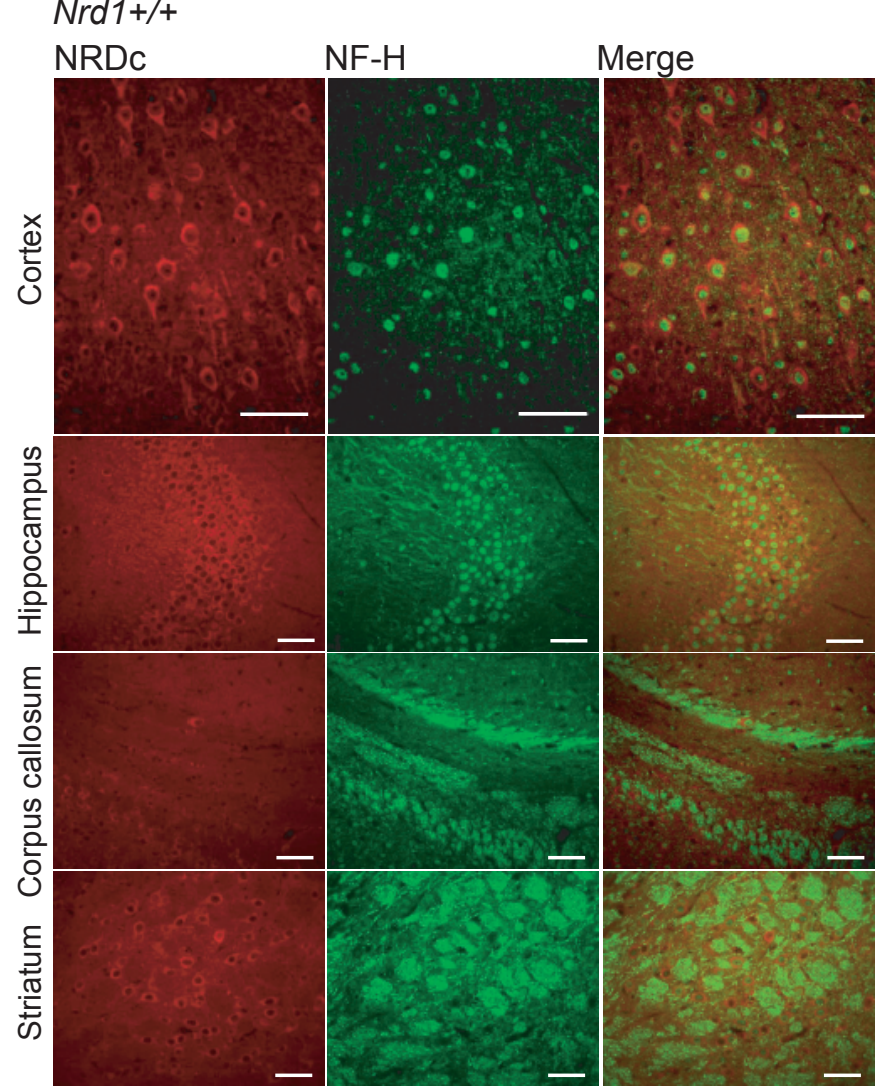

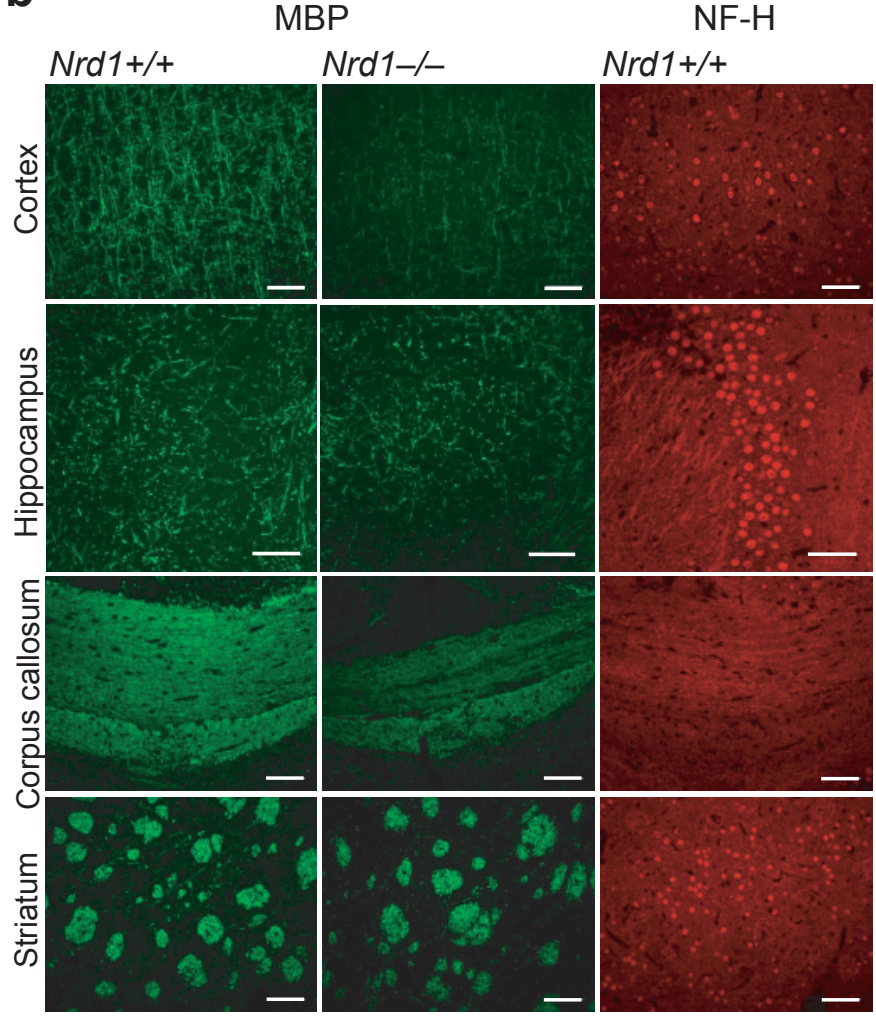

\section{Supplementary Figure 4}

Correlation between the expression level of NRDc and the level of myelination defect in Nrd1-/mice.

a, Regional distribution of NRDc in wild-type mouse brain.

Immunohistochemistry using an anti-NRDc antibody (red) and anti-NF-H antibody (green) with sections from wild-type mice brain. NRDc is expressed in neurons and dendrites, but not clearly detected in glial cells. Note that NRDc is highly expressed in cortical neurons, but neurons in striatum express relatively low level of NRDc. Scale bars, $250 \mu \mathrm{m}$.

b, Myelination defect in various regions of Nrd1-/- brain. Immunohistochemistry using an anti-MBP antibody (green) and anti-NF-H antibody (red) with sections from Nrd1+/+ and Nrd1-/ mice brain. Reduction of MBP expression, the myelination defect, is not very obvious in striatum, compared with the defect in the cortex, hippocampus and corpus callosum of Nrd1-/- brain. Scale bar: $250 \mu \mathrm{m}$. 


\section{E18.5}

Olig2

Nissl
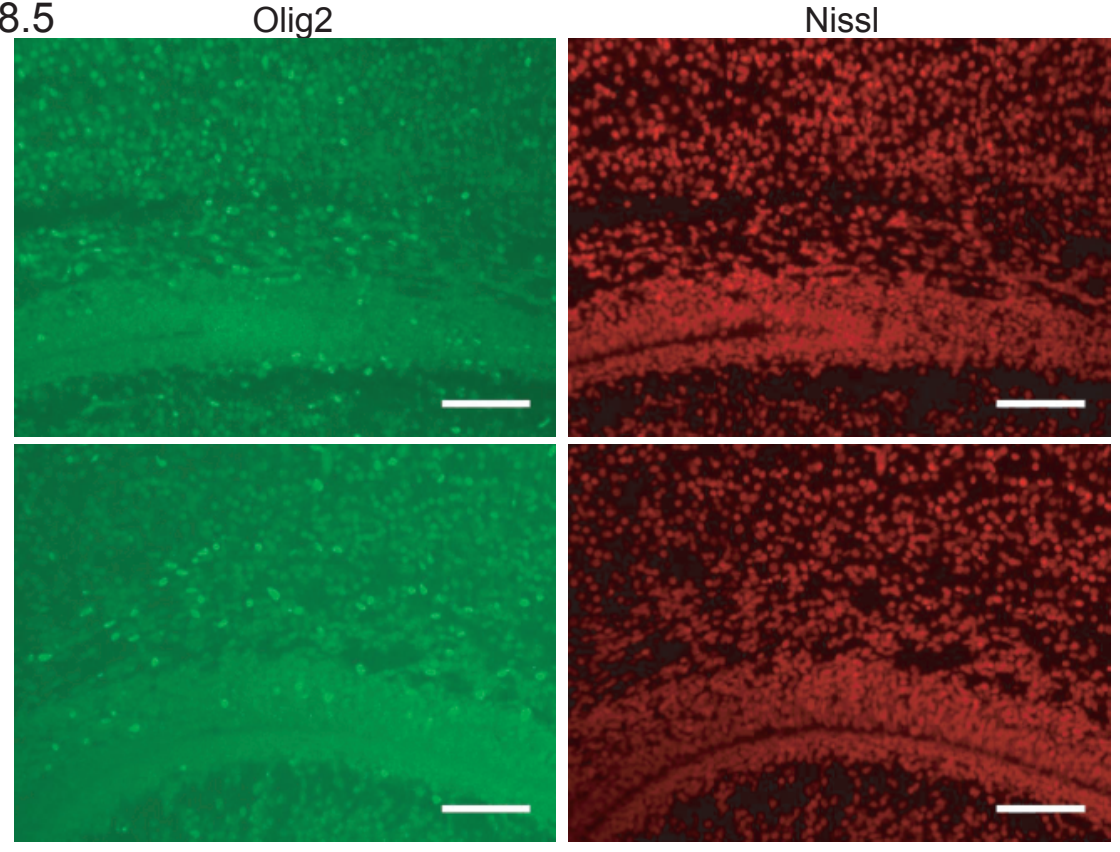

$\operatorname{Nrd1+/+}$

P5
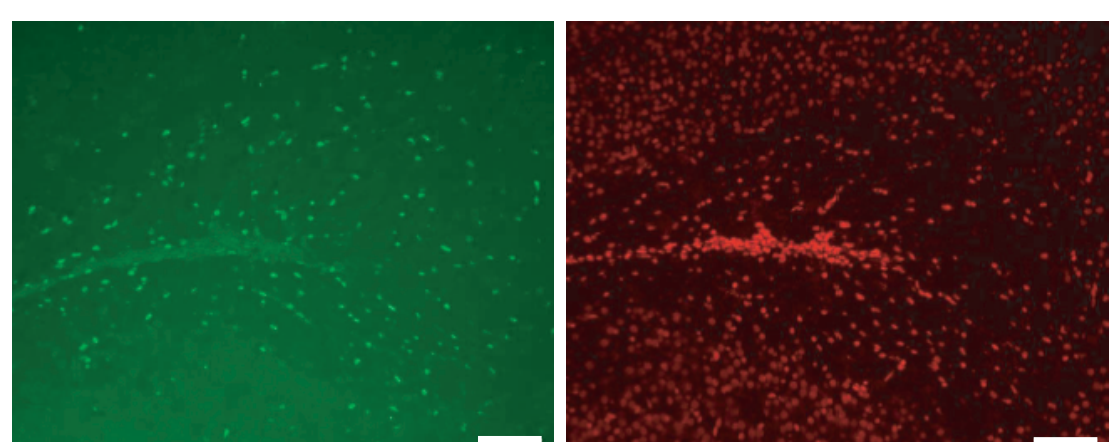

Nrd1-/-

$\operatorname{Nrd1+/+}$
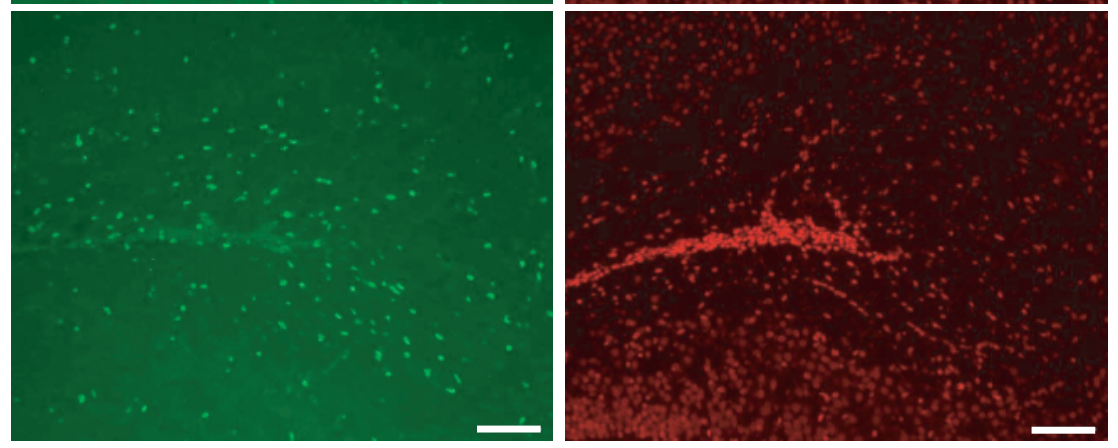

Nrd1-/-

b

E18.5

P5
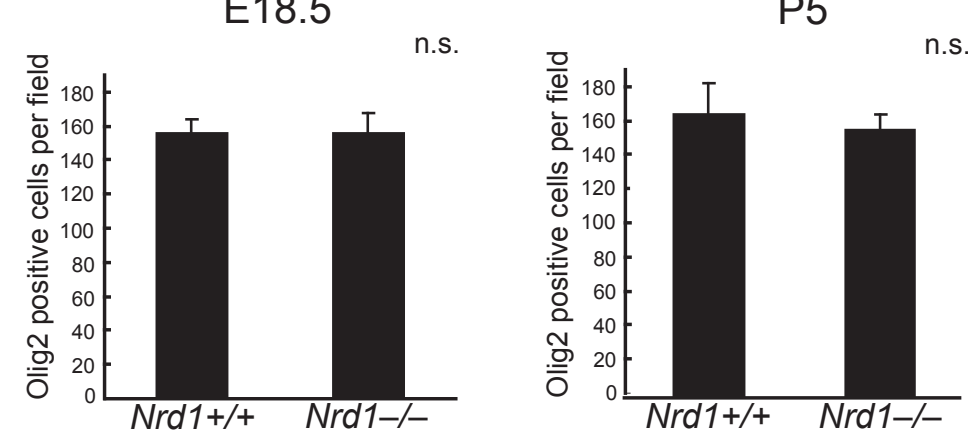

\section{Supplementary Figure 5}

No difference in the number of OPC between Nrd1+/+ and Nrd1-/- brains.

a, Immunohistochemistry using anti-Olig2 antibody in Nrd1+/+ and Nrd1-/- brains at E18.5 and P5. Scale bar; $250 \mu \mathrm{m}$.

b, Quantitative analysis of Olig2-positive cells demonstrated that there is no difference in the number of Olig2-positive cells between Nrd1+/+ and Nrd1-/- brains at E18.5 and P5.

Olig2-positive cells were counted at the same levels (20 non-overlapping fields / $n=2$ per genotype for each age). Data represent mean \pm s.e.m. 


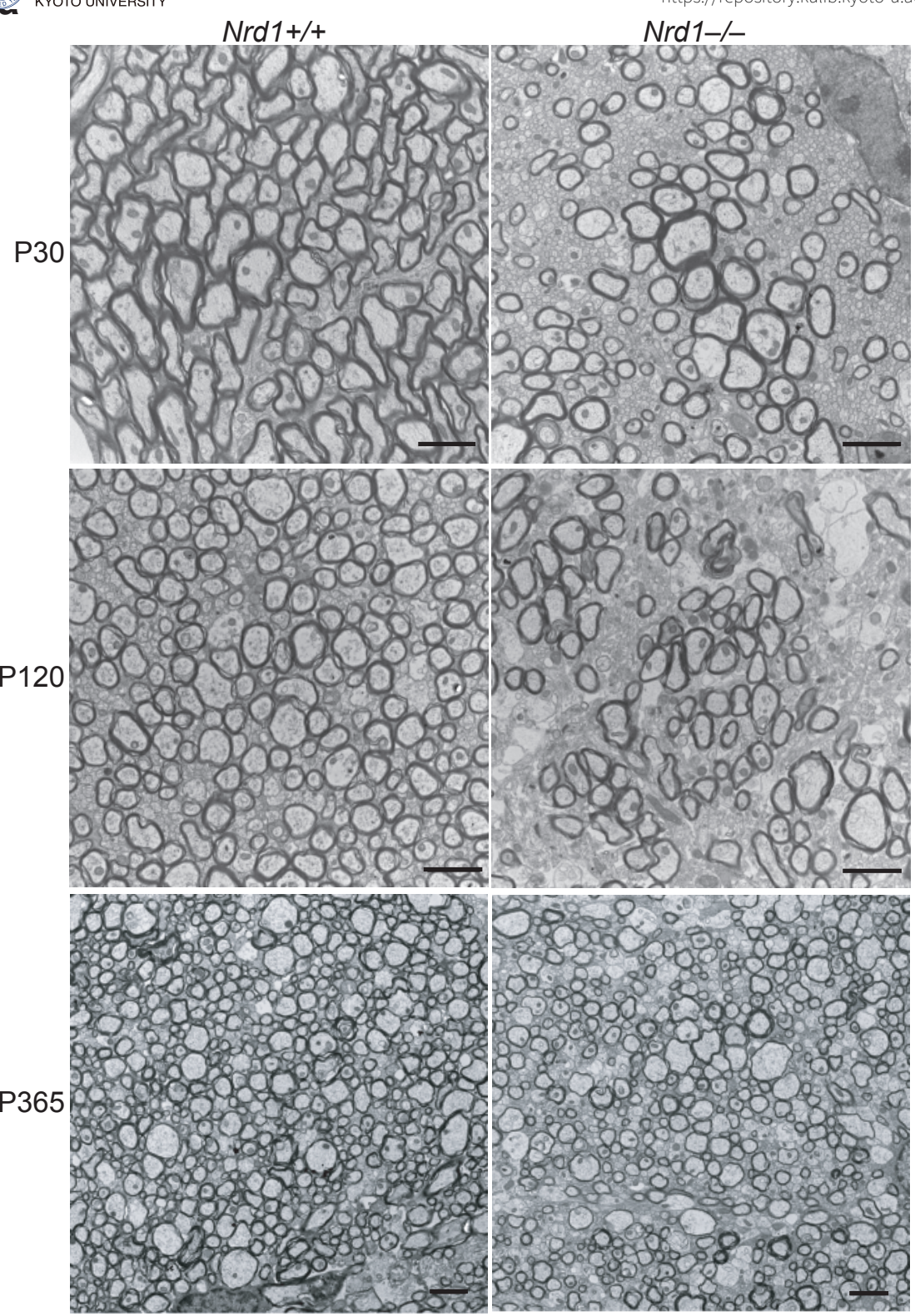

b

P30

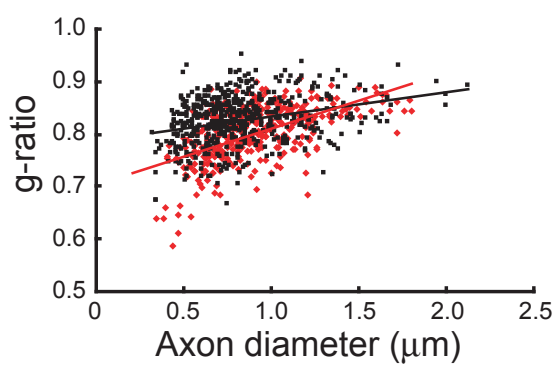

P120

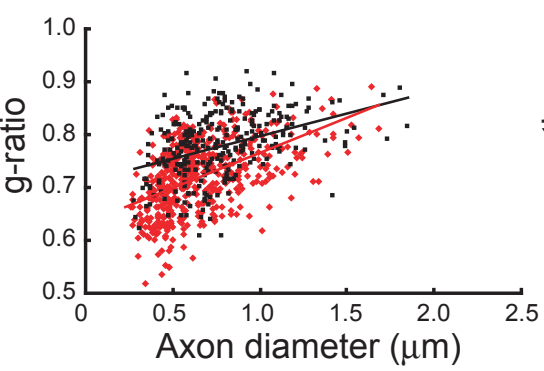

P365

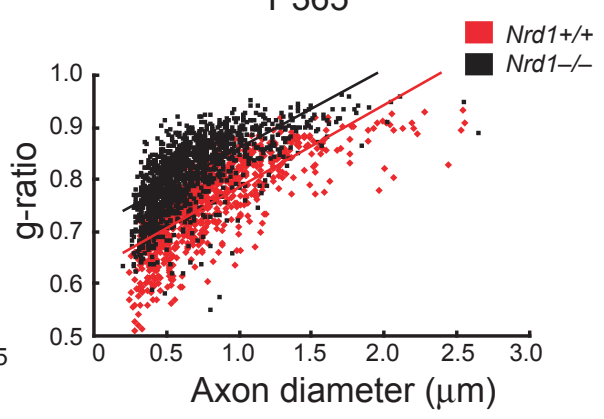

\section{Supplementary Figure 6}

Delay in the initiation of myelination and hypomyelination in Nrd1-/- mice.

a, Electron micrographs of transverse sections at the corpus callosum from Nrd1-/- mice (right) and Nrd1+/+ littermates (left) at P30 (upper), P120 (middle) and P365 (bottom). Scale bar, $2 \mu \mathrm{m}$.

b, Scatter plots of myelin thickness, expressed as g-ratios, against axon diameters at P30, P120 and P365 in Nrd1-/- (black) and wild-type (red) corpus callosum ( $n=2$ in each genotype for each age). No fewer than 500 myelinated fibers from each group were analyzed.

Average myelin sheath thickness (g-ratio) of myelinated fibers is shown in Figure $3 \mathrm{~d}$. 
京都大学

KYOTO UNIVERSTIY

Wire-hang test $p=0.1211$

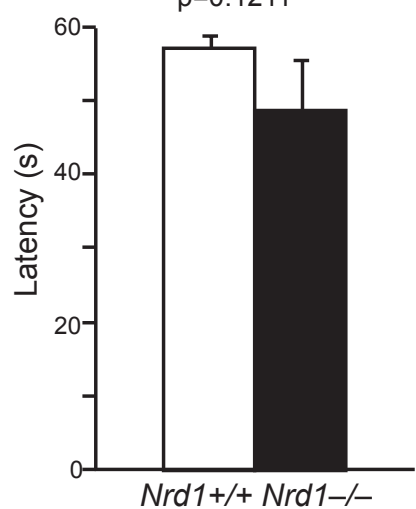

C

T-maze latency

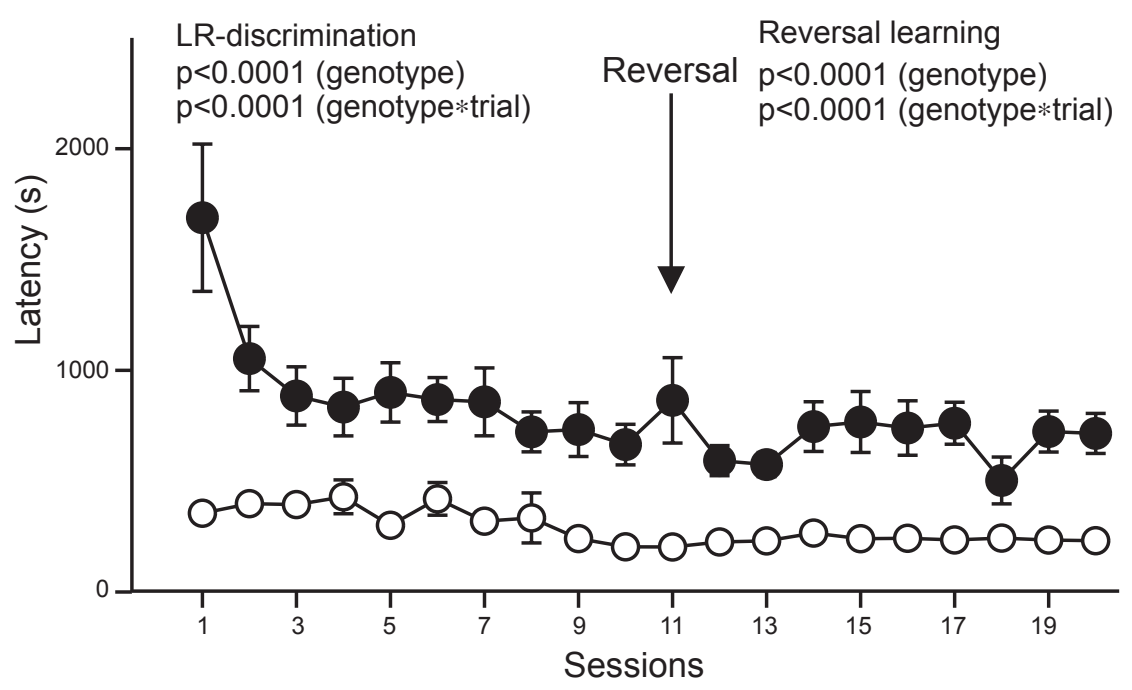

Hot plate test

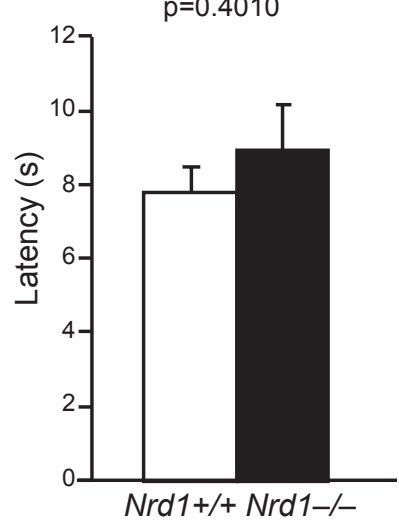

\section{Nrd1+/+}

- Nrd1-/

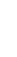

b

A Self-archived copy in
Kyoto University Research Information Repository

\section{Supplementary Figure 7}

Behavioral tests (Supplement for Figure 7).

a, Wire-hang test showed no significant difference between $\mathrm{Nrd1+/+}$ and $\mathrm{Nrd1}-/ /$ mice.

b. Hot plate test showed no significant difference in nociception or sensitivity to a paiful stimulus between $\mathrm{Nrd1}+/+$ and $\mathrm{Nrd1} 1-/$ mice.

c, Latency to complete session in the T-maze L-R discrimination task. Nrd1-/- mice took much longer to finish a session than did $\mathrm{Nrd1+/+}$ mice, most likely due to their slow walking. Age-matched littermates, $\mathrm{n}=12 ; \mathrm{Nrd1}+/+, \mathrm{n}=6$; Nrd1-/-.

The genotype effect was analyzed by a two-way repeated ANOVA in all tests. 


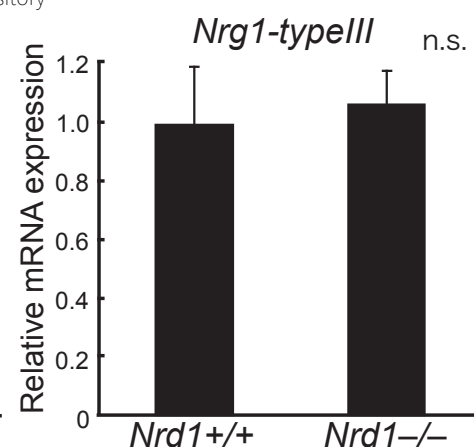

\section{Supplementary Figure 8}

Bace1 and Nrg1 mRNA expression in brain.

Bace1, Nrg1-typel and Nrg1-typelll mRNA levels in Nrd1+/+ and Nrd1-/- brains (P5; $n=3$ for each genotype) were analyzed by real-time PCR.

Expression level of each mRNA was standardized by that of $\beta$-actin, and the ratio was arbitrarily set at 1 in $\mathrm{Nrd1+/+}$.

There were no differences in the mRNA levels between $\mathrm{Nrd1+/+}$ and $\mathrm{Nrd1} 1-/$ brains. Data represent mean \pm s.e.m. 
京都大学

KYOTO UNIVERSITY blots for Figure 1a

Left: brain

Blot: NRDc

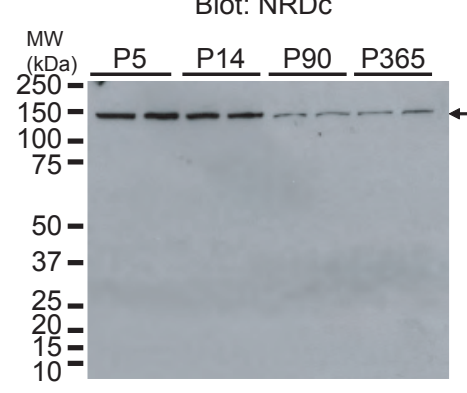

Blot: GAPDH

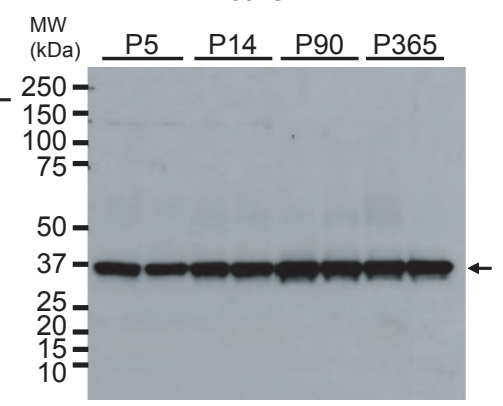

Center: brain Blot: NRDc, GAPDH

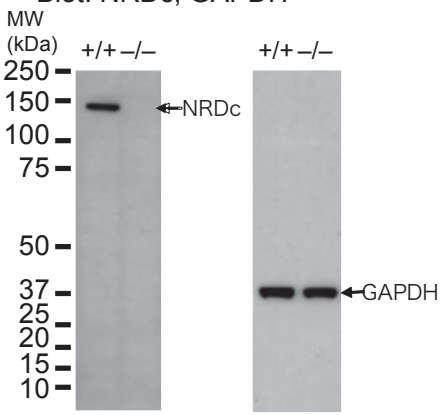

Right: MEF

Blot: NRDc, GAPDH MW

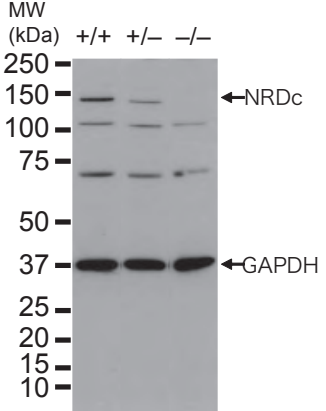

\section{Full-length blots for Figure $\mathbf{2 g}$}
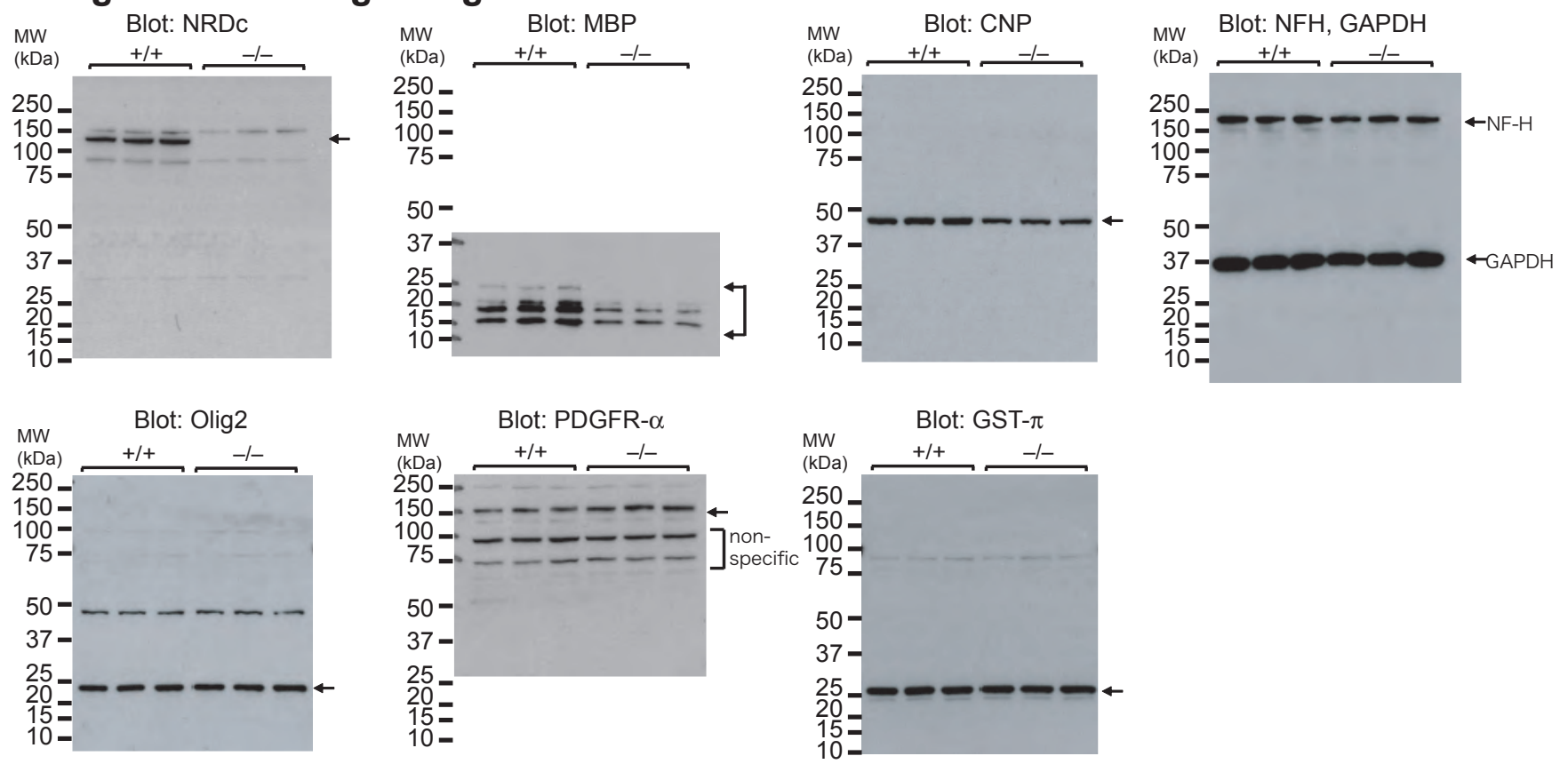

\section{Full-length blots for Figure $\mathbf{5 f}$}
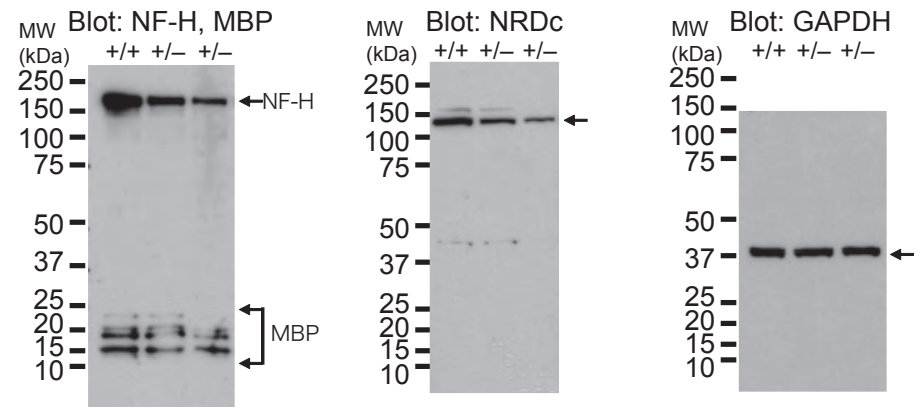

\section{Full-length blots for Figure 6e}

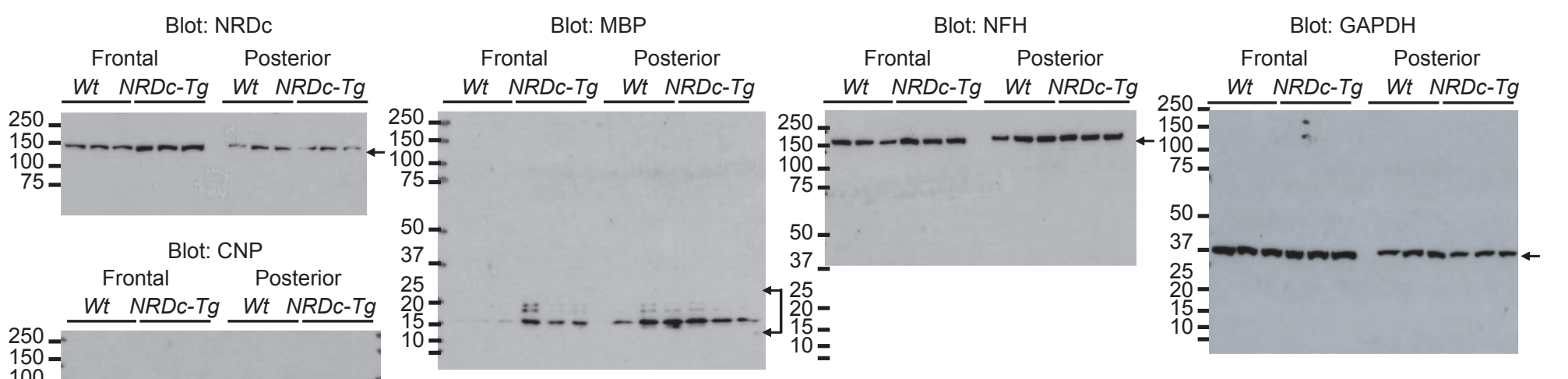

